\title{
Error analysis of an augmented mixed method for the Navier-Stokes problem with mixed boundary conditions
}

\author{
JESSIKA CAMAÑO† \\ Departamento de Matemática y Física Aplicadas, Universidad Católica de la Santísima \\ Concepción, Casilla 297, Concepción, Chile; and Centro de Investigación en Ingeniería \\ Matemática (CI $\left.{ }^{2} \mathrm{MA}\right)$, Universidad de Concepción, Casilla 160-C, Concepción, Chile

\section{RICARDO OYARZÚA} \\ GIMNAP, Departamento de Matemática, Universidad del Bío-Bío, Casilla 5-C, Concepción, \\ Chile; and Centro de Investigación en Ingeniería Matemática $\left(\mathrm{CI}^{2} \mathrm{MA}\right)$, Universidad de \\ Concepción, Casilla 160-C, Concepción, Chile \\ RICARDO RUIZ-BAIER $\S$ \\ Mathematical Institute, Oxford University, Woodstock Road, OX2 6GG Oxford, UK \\ AND \\ GIORDANO TIERRA \\ Department of Mathematics, Temple University, Philadelphia, PA 19122, USA
}

[Received on December 10, 2016; revised on June 19, 2017]

\begin{abstract}
In this paper we analyze an augmented mixed finite element method for the steady Navier-Stokes equations. More precisely, we extend the recent results from Camaño et al. (2017) to the case of mixed no-slip and traction) boundary conditions in different parts of the boundary, and introduce and analyze a new pseudostress-velocity augmented mixed formulation for the fluid flow problem. The well-posedness analysis is carried out by combining the classical Babuška-Brezzi theory and Banach's fixed-point Theorem. A proper adaptation of the arguments exploited in the continuous analysis allows us to state suitable hypotheses on the finite element subspaces ensuring that the associated Galerkin scheme is well-defined. For instance, Raviart-Thomas elements of order $k \geqslant 0$ and continuous piecewise polynomials of degree $k+1$ for the nonlinear pseudo-stress tensor and velocity, respectively, yield optimal convergence rates. In addition, we derive a reliable and efficient residual-based a posteriori error estimator for the proposed discretization. The proof of reliability hinges on the global inf-sup condition and the local approximation properties of the Clément interpolant, whereas the efficiency of the estimator follows from a inverse inequalities and localization via edge-bubble functions. A set of numerical results exemplifies the performance of the augmented method with mixed boundary conditions. The tests also confirm the reliability and efficiency of the estimator, and show the performance of the associated adaptive algorithm.
\end{abstract}

Keywords: Navier-Stokes, mixed finite element method, augmented formulation, mixed boundary conditions, Raviart-Thomas elements, a posteriori error analysis.

Mathematical subject classification 2000: . 65N30, 65N12, 65N15, 76R05, 76D07.

\section{Introduction}

This paper is concerned with the numerical study of the Navier-Stokes problem with Dirichlet and traction boundary conditions in different parts of the boundary. Our focus will be on an augmented mixed pseudostressbased formulation of this problem.

\footnotetext{
${ }^{\dagger}$ Email: jecamano@ucsc.cl

¥Corresponding author. Email: royarzua@ubiobio.cl

§mail: ruizbaier@maths.ox.ac.uk

๑Email: gtierra@temple.edu
}

(c) The author 2017. Published by Oxford University Press on behalf of the Institute of Mathematics and its Applications. All rights reserved. 
An important body of recent literature deals with numerical techniques to numerically solve the NavierStokes equations. A rich variety of methods is available, having different features being tested for many fundamental cases and industrial applications. Among these methods, we give special attention to numerical schemes based on dual-mixed formulations, which have gained considerable attention (already over the last few decades) mainly due to the fact that, on the one hand, they allow to unify the analysis for Newtonian and non-Newtonian flows, and on the other hand, they permit to approximate additional variables of interest as a simple post-processing from the primal unknowns (see for instance Camaño et al. 2017. 2016, Cai \& Zhang. 2012, Farhloul et al. 2009, Howell \& Walkington 2013 and the references therein). For example, dual-mixed methods based on stresses or pseudostress tensors has proven remarkable success, especially in coupled problems such as Stokes-Darcy, Navier-Stokes/Darcy, and Boussinesq systems (see, for instance Caucao et al. 2015, Colmenares et al. 2016, Gatica et al. 2011b 2012).

Regarding Navier-Stokes equations, Cai \& Zhang (2012) introduced and analyzed a conforming dualmixed method where the pseudostress, the velocity and the pressure are the main unknowns of the system (see also Cai et al. 2010). Their formulation accommodates the use of Raviart-Thomas elements for the pseudostress, yielding approximations with accuracy $O\left(h^{k+1-n / 6}\right)(n=2,3)$ in the $L^{3}$-norm. More recently, Camaño et al. (2017) proposed a new pseudostress-based mixed method for the pure Dirichlet Navier-Stokes problem. Such a method involves a pseudostress tensor depending nonlinearly on the velocity through the convective term, whereas pressure is eliminated via the incompressibility constraint, and can be later recovered as a postprocess of the pseudostress tensor. The formulation from Camaño et al. (2017) results in RaviartThomas approximations of pseudostresses exhibiting optimal convergence. Up to the authors' knowledge, that is the first Raviart-Thomas-based mixed method for Navier-Stokes providing optimal convergence for all the unknowns, and the key feature behind this optimality is the incorporation of residual terms arising from the constitutive and equilibrium equations. This (so-called augmentation) procedure allows to circumvent inf-sup conditions, and consequently relaxing the hypotheses on the discrete subspaces. Further details on augmented approaches can be found in e.g. Brezzi \& Fortin (1991); Franca \& Hughes (1988); Gatica (2006) (see also Howell \& Walkington, 2013, Farhloul et al. 2009).

The purpose of the present work is to extend the results in Camaño et al.(2017) to the case of mixed boundary conditions, particularly useful, when modelling free surface flow or requiring outflow/artificial boundaries (see e.g. Bänsch, 2001 2014, Bruneat \& Fabrie, 2001; Dong \& Shen, 2015). In this regard, the only contribution addressing dual-mixed formulations and mixed boundary conditions for Navier-Stokes, is Farhloul et al. (2008). The authors study a strain-velocity-vorticity-pressure formulation, and use the discrete elements developed in Farhloul \& Fortin (1997) (designed for Stokes and elasticity problems), to obtain a quasi-optimal mixed method.

Here, and similarly to Camaño et al. (2017), the main unknowns are the velocity and a pseudostress tensor relating the strain tensor with the convective term. The pressure is eliminated using the fluid incompressibility, and can be recovered as a postprocess of the pseudostress. Moreover, due to the convective term, the velocity is sought in $\mathrm{H}^{1}$, which requires augmentation via Galerkin terms arising from the constitutive and equilibrium equations. In contrast with the analysis for the pure Dirichlet case in Camaño et al. (2017), here the mixed boundary conditions imply that the resulting augmented variational problem is written as a saddle-point problem. Therefore we can employ the classical Babuška-Brezzi theory and Banach's fixed point theorem to prove existence and uniqueness of solution to both the continuous and discrete problems. In addition, for generic finite dimensional spaces we derive a suitable Céa's estimate; and choosing for instance Raviart-Thomas elements of degree $k$ for the pseudostress tensor, and continuous piecewise polynomial elements of degree $k+1$ for the velocity, provides optimal rates of convergence. This is one of the principal advantages of our method. We highlight that, differently from previous results related with dual-mixed formulations for elliptic problems dealing with strain tensors and Neumann-type boundary conditions (see for instance Camaño et al. 2015 , Gatica. 2006), in this paper we do not introduce the vorticity (or rotation in the case of the elasticity problem) nor vectorial or scalar Lagrange multipliers on the boundary as further unknowns to derive our formulation, avoiding hence the introduction of costly unknowns to the system.

Additionally to the continuous and discrete analyses of this paper, and in order to guarantee optimal convergence even under the eventual presence of boundary layers and singularities, we derive a reliable and efficient, residual-based, a posteriori error estimator for our method. For the proof of reliability, instead of using a classical Helmholtz decomposition (see its application in similar contexts in e.g. Camaño et al. 2015, Gatica et al. 2016), here we use the global inf-sup condition, integration by parts, and the approximation properties of the Clément interpolator. This approach yields a reduction in the number of terms composing the error indicator, it makes the analysis simpler; and it unifies the analysis for the 2D and 3D cases. The proof of efficiency of the estimator consists of a localization method based on edge-bubble (or face-bubble functions) and inverse inequalities. 
The rest of this paper is organized as follows. In Section 2 we introduce the model problem and rewrite the equations in terms of the pseudostress tensor and the velocity. In Section 3 we derive the augmented variational formulation and prove its well-posedness by means of a fixed-point strategy and the classical Babuška-Brezzi theory. In Section 4 we define the Galerkin scheme and derive suitable hypotheses on the finite element subspaces ensuring that the discrete scheme becomes well-posed and the corresponding Céa's estimate holds. We also describe a specific choice of finite element subspaces, namely Raviart-Thomas of order $k$ and continuous piecewise polynomials of degree $k+1$ and derive the corresponding theoretical rate of convergence. In Section 5 we introduce the a posteriori error estimator and prove its reliability and efficiency. Finally, several numerical examples employing the spaces introduced in Section 4 illustrating the accuracy of the method, and showing the good performance of the associated adaptive algorithm are reported in Section 6

We end this section by recalling some definitions and fixing useful notations. Given the vector fields $\mathbf{v}=\left(v_{i}\right)_{i=1, n}$ and $\mathbf{w}=\left(w_{i}\right)_{i=1, n}$, with $n \in\{2,3\}$, we set the gradient, divergence, and tensor product operators, by

$$
\nabla \mathbf{v}:=\left(\frac{\partial v_{i}}{\partial x_{j}}\right)_{i, j=1, n}, \quad \operatorname{div} \mathbf{v}:=\sum_{j=1}^{n} \frac{\partial v_{j}}{\partial x_{j}}, \quad \text { and } \quad \mathbf{v} \otimes \mathbf{w}:=\left(v_{i} w_{j}\right)_{i, j=1, n} .
$$

Furthermore, for any tensor fields $\mathbf{S}:=\left(S_{i j}\right)_{i, j=1, n}$ and $\mathbf{R}:=\left(R_{i j}\right)_{i, j=1, n}$, we define the transpose, the trace, the tensor inner product, and the deviatoric tensor, respectively, by

$$
\mathbf{S}^{t}:=\left(S_{j i}\right)_{i, j=1, n}, \quad \operatorname{tr}(\mathbf{S}):=\sum_{i=1}^{n} S_{i i}, \quad \mathbf{S}: \mathbf{R}:=\sum_{i, j=1}^{n} S_{i j} R_{i j}, \quad \text { and } \quad \mathbf{S}^{\mathrm{d}}:=\mathbf{S}-\frac{1}{n} \operatorname{tr}(\mathbf{S}) \mathrm{I},
$$

where $\mathrm{I}$ is the identity matrix in $\mathbb{R}^{n \times n}$. When no confusion arises, $|\cdot|$ will denote the Euclidean norm in $\mathbb{R}^{n}$ or $\mathbb{R}^{n \times n}$. Additionally, we will utilize standard simplified terminology for Sobolev spaces and norms. In particular, if $\mathscr{O}$ is a domain, $\Gamma$ is an open or closed Lipschitz curve (respectively surface in $\mathbb{R}^{3}$ ), and $r \in \mathbb{R}$, we define

$$
\mathbf{H}^{r}(\mathscr{O}):=\left[\mathrm{H}^{r}(\mathscr{O})\right]^{n}, \quad \mathbb{H}^{r}(\mathscr{O}):=\left[\mathrm{H}^{r}(\mathscr{O})\right]^{n \times n}, \quad \text { and } \quad \mathbf{H}^{r}(\Gamma):=\left[\mathrm{H}^{r}(\Gamma)\right]^{n},
$$

and adopt the usual convention of writing $\mathbf{L}^{2}(\mathscr{O}), \mathbb{L}^{2}(\mathscr{O})$, and $\mathbf{L}^{2}(\Gamma)$ instead of $\mathbf{H}^{0}(\mathscr{O}), \mathbb{H}^{0}(\mathscr{O})$, and $\mathbf{H}^{0}(\Gamma)$, respectively. The corresponding norms are denoted by $\|\cdot\|_{r, \mathscr{O}}$ for $\mathrm{H}^{r}(\mathscr{O}), \mathbf{H}^{r}(\mathscr{O})$ and $\mathbb{H}^{r}(\mathscr{O})$, and $\|\cdot\|_{r, \Gamma}$ for $\mathrm{H}^{r}(\Gamma)$ and $\mathbf{H}^{r}(\Gamma)$. We also write $|\cdot|_{r, \mathscr{O}}$ for the $\mathbf{H}^{r}$-seminorm. In addition, we recall that

$$
\mathbf{H}(\operatorname{div} ; \mathscr{O}):=\left\{\mathbf{w} \in \mathbf{L}^{2}(\mathscr{O}): \quad \operatorname{div} \mathbf{w} \in L^{2}(\mathscr{O})\right\}
$$

is a standard Hilbert space (see, e.g. Brezzi \& Fortin 1991, Girault \& Raviart 1986), and the space of matrix valued functions whose rows belong to $\mathbf{H}(\operatorname{div} ; \mathscr{O})$ will be denoted by $\mathbb{H}(\operatorname{div} ; \mathscr{O})$. The norms of $\mathbf{H}(\operatorname{div} ; \mathscr{O})$ and $\mathbb{H}(\mathbf{d i v} ; \mathscr{O})$ are denoted by $\|\cdot\|_{\operatorname{div}, \mathscr{O}}$ and $\|\cdot\|_{\operatorname{div}, \mathscr{O}}$, respectively. Note also that $\mathbb{H}(\mathbf{d i v} ; \mathscr{O})$ can be characterized as the space of matrix valued functions $\mathbf{S}$ such that $\mathbf{c}^{t} \mathbf{S} \in \mathbf{H}(\operatorname{div} ; \mathscr{O})$ for any constant column vector $\mathbf{c}$. In addition, it is easy to see that there holds:

$$
\mathbb{H}(\operatorname{div} ; \mathscr{O})=\mathbb{H}_{0}(\operatorname{div} ; \mathscr{O}) \oplus P_{0}(\mathscr{O}) \mathrm{I}
$$

where

$$
\mathbb{H}_{0}(\operatorname{div} ; \mathscr{O}):=\left\{\mathbf{S} \in \mathbb{H}(\operatorname{div} ; \mathscr{O}): \quad \int_{\mathscr{O}} \operatorname{tr} \mathbf{S}=0\right\}
$$

and $P_{0}(\mathscr{O})$ is the space of constant polynomials on $\mathscr{O}$. More precisely, each $\mathbf{S} \in \mathbb{H}(\mathbf{d i v} ; \mathscr{O})$ can be decomposed uniquely as:

$$
\mathbf{S}=\mathbf{S}_{0}+c \mathbf{I}, \quad \text { with } \quad \mathbf{S}_{0} \in \mathbb{H}_{0}(\mathbf{d i v} ; \mathscr{O}) \quad \text { and } \quad c:=\frac{1}{n|\mathscr{O}|} \int_{\mathscr{O}} \operatorname{tr} \mathbf{S} \in \mathbb{R}
$$

Such a decomposition will be exploited in the subsequent analysis of weak formulations.

Furthermore, given an integer $k \geqslant 0$ and a set $M \subseteq \mathbb{R}^{n}, P_{k}(M)$ denotes the space of polynomials on $M$ of degree $\leqslant k$. In addition, we set $\mathbf{P}_{k}(M):=\left[P_{k}(M)\right]^{n}$ and $\mathbb{P}_{k}(M):=\left[P_{k}(M)\right]^{n \times n}$. Finally, throughout the rest of the paper, we employ $\mathbf{0}$ to denote a generic null vector (including the null functional and operator), and use $C$ and $c$, with or without subscripts, bars, tildes or hats, to denote generic constants independent of the discretization, which may take different values at different places. 


\section{The model problem}

Let $\Omega \subseteq \mathbb{R}^{n}$ be a bounded domain, $n \in\{2,3\}$, with Lipschitz-boundary $\Gamma$. The boundary of this domain is divided into two portions $\Gamma_{D}$ and $\Gamma_{N}$, such that $\bar{\Gamma}_{D} \cup \bar{\Gamma}_{N}=\Gamma$ and $\left|\Gamma_{D}\right|>0$ on which different types of boundary conditions will be imposed. We consider the flow of an incompressible fluid with constant viscosity, where the Cauchy stress of the fluid $\boldsymbol{\sigma}$ can be written as

$$
\boldsymbol{\sigma}:=2 v \mathbf{e}(\mathbf{u})-p \mathbf{I},
$$

with $\mathbf{u}$ denoting the velocity field, $v$ the viscosity, $p$ the pressure and $\mathbf{e}(\mathbf{u})$ the strain rate

$$
\mathbf{e}(\mathbf{u}):=\frac{1}{2}\left(\nabla \mathbf{u}+(\nabla \mathbf{u})^{t}\right) .
$$

The set of governing equations consists on the incompressible steady-state Navier-Stokes problem with constant viscosity and mixed boundary conditions, given by

$$
\begin{gathered}
(\mathbf{u} \cdot \nabla) \mathbf{u}-\operatorname{div} \boldsymbol{\sigma}=\mathbf{f} \text { in } \Omega, \quad \operatorname{div} \mathbf{u}=0 \quad \text { in } \Omega, \\
\mathbf{u}=\mathbf{0} \text { on } \Gamma_{D}, \quad \boldsymbol{\sigma} \mathbf{n}=\mathbf{g} \text { on } \Gamma_{N},
\end{gathered}
$$

where $\mathbf{f}$ and $\mathbf{g}$ are given data living in spaces to be specified next and $\mathbf{n}$ is the outward unit vector normal to the boundary $\Gamma_{N}$.

We proceed analogously as in Camaño et al. (2017) and introduce the nonlinear-pseudostress tensor

$$
\mathbf{T}:=\boldsymbol{\sigma}-\mathbf{u} \otimes \mathbf{u}=2 v \mathbf{e}(\mathbf{u})-p \mathrm{I}-\mathbf{u} \otimes \mathbf{u},
$$

which assembles the Cauchy stress tensor $\boldsymbol{\sigma}$ with the convective term $\mathbf{u} \otimes \mathbf{u}$. Note that this tensor represents the flux associated to the momentum density. Then, using the incompressibility condition $\operatorname{div} \mathbf{u}=\operatorname{tr}(\mathbf{e}(\mathbf{u}))=0$ in $\Omega$, we can easily obtain the identities (Camaño et al. 2017. Sect. 2.1)

$$
p=-\frac{1}{n}(\operatorname{tr}(\mathbf{T})+\operatorname{tr}(\mathbf{u} \otimes \mathbf{u})) \quad \text { in } \quad \Omega \quad \text { and } \quad-\operatorname{div} \mathbf{T}=\mathbf{f} \quad \text { in } \quad \Omega .
$$

In particular, using the first identity above we can eliminate the pressure from 2.3 to obtain

$$
\mathbf{T}^{\mathrm{d}}=2 v \mathbf{e}(\mathbf{u})-(\mathbf{u} \otimes \mathbf{u})^{\mathrm{d}} \quad \text { in } \quad \Omega .
$$

In turn, noticing that $\mathbf{e}(\mathbf{u})=\nabla \mathbf{u}-\boldsymbol{\omega}(\mathbf{u})$, with

$$
\boldsymbol{\omega}(\mathbf{u}):=\frac{1}{2}\left(\nabla \mathbf{u}-(\nabla \mathbf{u})^{t}\right)
$$

we have that 2.5 becomes

$$
\mathbf{T}^{\mathrm{d}}=2 v \nabla \mathbf{u}-2 v \boldsymbol{\omega}(\mathbf{u})-(\mathbf{u} \otimes \mathbf{u})^{\mathrm{d}} \quad \text { in } \quad \Omega .
$$

It is then readily seen that 2.2) can be rewritten in terms of $\mathbf{T}$ and $\mathbf{u}$ as follows

$$
\begin{gathered}
\mathbf{T}^{\mathrm{d}}=2 v \nabla \mathbf{u}-2 v \boldsymbol{\omega}(\mathbf{u})-(\mathbf{u} \otimes \mathbf{u})^{\mathrm{d}} \quad \text { in } \quad \Omega, \quad-\operatorname{div} \mathbf{T}=\mathbf{f} \quad \text { in } \quad \Omega, \\
\mathbf{T}=\mathbf{T}^{t} \quad \text { in } \quad \Omega, \quad \mathbf{u}=\mathbf{0} \quad \text { on } \quad \Gamma_{D} \quad \text { and } \quad \mathbf{T n}+(\mathbf{u} \otimes \mathbf{u}) \mathbf{n}=\mathbf{g} \quad \text { on } \quad \Gamma_{N} .
\end{gathered}
$$

Similarly to Camaño et al. (2017), in what follows we make use of (2.8) to derive our variational formulation.

REMARK 2.1 It is well-known that when considering mixed boundary conditions, the constitutive equation 2.1) must be written in terms of the strain rate $\mathbf{e}(\mathbf{u})$ (see Farhloul et al. 2008), and therefore integration by parts cannot be performed straightforwardly. A common remedy is to introduce a further unknown to the system, say $\gamma=\frac{1}{2}\left(\nabla \mathbf{u}-(\nabla \mathbf{u})^{t}\right)$, and utilize the decomposition $\mathbf{e}(\mathbf{u})=\nabla \mathbf{u}+\gamma$, similarly as we do in this work (see for instance Arnold et al. (1984b); Farhloul et al. (2008); Gatica (2006)). However, here we take advantage of the fact that $\mathbf{u}$ is sought in $\mathbf{H}^{1}$, and we keep $\mathbf{T}$ and $\mathbf{u}$ as the only unknowns of the problem, avoiding the necessity of further unknowns in the system (which would result in an increased computational cost).

\section{Continuous problem}

In this section we introduce our augmented mixed variational formulation and address its solvability. We begin by defining the variational problem. 


\subsection{The augmented mixed variational problem}

We now turn to the derivation of the weak formulation of (2.8). We start by multiplying the first equation of 2.8) by a suitable test function $\mathbf{S} \in \mathbb{H}(\mathbf{d i v} ; \Omega)$, and then integrating by parts, employing the Dirichlet boundary condition $\mathbf{u}=\mathbf{0}$ on $\Gamma_{D}$, and using the identities

$$
\mathbf{T}^{\mathrm{d}}: \mathbf{S}=\mathbf{T}^{\mathrm{d}}: \mathbf{S}^{\mathrm{d}} \quad \text { and } \quad(\boldsymbol{\omega}(\mathbf{u}), \mathbf{S})_{\Omega}=\frac{1}{2}(\operatorname{curl}(\mathbf{u}), \operatorname{as}(\mathbf{S}))_{\Omega},
$$

with

$$
\operatorname{curl}(\mathbf{v}):= \begin{cases}\frac{\partial v_{2}}{\partial x_{1}}-\frac{\partial v_{1}}{\partial x_{2}} & \text { in } \mathbb{R}^{2} \\ \nabla \times \mathbf{v}:=\left(\frac{\partial v_{3}}{\partial x_{2}}-\frac{\partial v_{2}}{\partial x_{3}}, \frac{\partial v_{1}}{\partial x_{3}}-\frac{\partial v_{3}}{\partial x_{1}}, \frac{\partial v_{2}}{\partial x_{1}}-\frac{\partial v_{1}}{\partial x_{2}}\right) & \text { in } \mathbb{R}^{3}\end{cases}
$$

and

$$
\operatorname{as}(\mathbf{S}):= \begin{cases}S_{21}-S_{12} & \text { in } \mathbb{R}^{2}, \\ \left(S_{32}-S_{23}, S_{13}-S_{31}, S_{21}-S_{12}\right) & \text { in } \mathbb{R}^{3},\end{cases}
$$

we readily obtain

$$
\left(\mathbf{T}^{\mathrm{d}}, \mathbf{S}^{\mathrm{d}}\right)_{\Omega}+2 v(\mathbf{u}, \operatorname{div} \mathbf{S})_{\Omega}+v(\operatorname{curl}(\mathbf{u}), \operatorname{as}(\mathbf{S}))_{\Omega}+\left((\mathbf{u} \otimes \mathbf{u})^{\mathrm{d}}, \mathbf{S}\right)_{\Omega}-2 v\langle\mathbf{S n}, \mathbf{u}\rangle_{\Gamma_{N}}=0,
$$

Above, the symbol $\langle\cdot, \cdot\rangle_{\Gamma_{N}}$ represents the duality paring of $\mathbf{H}_{00}^{-1 / 2}\left(\Gamma_{N}\right)$ and $\mathbf{H}_{00}^{1 / 2}\left(\Gamma_{N}\right)$ with respect to the $\mathbf{L}^{2}(\Gamma)$ inner product, where

$$
\mathbf{H}_{00}^{1 / 2}\left(\Gamma_{N}\right):=\left\{\left.\mathbf{v}\right|_{\Gamma_{N}}: \mathbf{v} \in \mathbf{H}_{\Gamma_{D}}^{1}(\Omega)\right\} \quad \text { and } \quad \mathbf{H}_{00}^{-1 / 2}\left(\Gamma_{N}\right)=\left[\mathbf{H}_{00}^{1 / 2}\left(\Gamma_{N}\right)\right]^{\prime},
$$

with

$$
\mathbf{H}_{\Gamma_{D}}^{1}(\Omega):=\left\{\mathbf{v} \in \mathbf{H}^{1}(\Omega):\left.\mathbf{v}\right|_{\Gamma_{D}}=\mathbf{0}\right\} .
$$

In what follows, the norms of $\mathbf{H}_{00}^{1 / 2}\left(\Gamma_{N}\right)$ and $\mathbf{H}_{00}^{-1 / 2}\left(\Gamma_{N}\right)$ are denoted by $\|\cdot\|_{1 / 2,00, \Gamma_{N}}$ and $\|\cdot\|_{-1 / 2,00, \Gamma_{N}}$, respectively. In particular, we observe that if $E_{0}: \mathbf{H}^{1 / 2}\left(\Gamma_{N}\right) \rightarrow \mathbf{L}^{2}(\Gamma)$ is the extension operator defined by

$$
E_{0}(\psi):=\left\{\begin{array}{ll}
\psi & \text { on } \Gamma_{N}, \\
\mathbf{0} & \text { on } \Gamma_{D},
\end{array} \quad \forall \psi \in \mathbf{H}^{1 / 2}\left(\Gamma_{N}\right)\right.
$$

then, the space $\mathbf{H}_{00}^{1 / 2}\left(\Gamma_{N}\right)$ can be defined equivalently as

$$
\mathbf{H}_{00}^{1 / 2}\left(\Gamma_{N}\right):=\left\{\psi \in \mathbf{H}^{1 / 2}\left(\Gamma_{N}\right): E_{0}(\psi) \in \mathbf{H}^{1 / 2}(\Gamma)\right\}
$$

endowed with the norm $\|\psi\|_{1 / 2,00, \Gamma_{N}}=\left\|E_{0}(\psi)\right\|_{1 / 2, \Gamma}$, where

$$
\|\boldsymbol{\xi}\|_{1 / 2, \Gamma}:=\inf \left\{\|\mathbf{v}\|_{1, \Omega}: \mathbf{v} \in \mathbf{H}^{1}(\Omega) \text { such that }\left.\mathbf{v}\right|_{\Gamma}=\boldsymbol{\xi}\right\} .
$$

From the latter, it can be readily seen that

$$
\|\mathbf{v}\|_{1 / 2,00, \Gamma_{N}}=\|\mathbf{v}\|_{1 / 2, \Gamma} \leqslant\|\mathbf{v}\|_{1, \Omega} \quad \forall \mathbf{v} \in \mathbf{H}_{\Gamma_{D}}^{1}(\Omega) .
$$

On the other hand, since we are interested in deriving an augmented formulation as the one in Camaño et al. (2017), we now multiply the second equation of 2.8) by $\mathbf{v} \in \mathbf{H}_{\Gamma_{D}}^{1}(\Omega)$, and differently from Camaño et al. 2017), we integrate by parts and utilize and the boundary condition $\mathbf{T n}+(\mathbf{u} \otimes \mathbf{u}) \mathbf{n}=\mathbf{g}$ on $\Gamma_{N}$, to deduce

$$
(\mathbf{T}, \nabla \mathbf{v})_{\Omega}-\langle\mathbf{g}, \mathbf{v}\rangle_{\Gamma_{N}}+\langle(\mathbf{u} \otimes \mathbf{u}) \mathbf{n}, \mathbf{v}\rangle_{\Gamma_{N}}=(\mathbf{f}, \mathbf{v})_{\Omega},
$$

which owing to the symmetry of $\mathbf{T}$ can be rewritten as

$$
(\mathbf{T}, \mathbf{e}(\mathbf{v}))_{\Omega}+\langle(\mathbf{u} \otimes \mathbf{u}) \mathbf{n}, \mathbf{v}\rangle_{\Gamma_{N}}=(\mathbf{f}, \mathbf{v})_{\Omega}+\langle\mathbf{g}, \mathbf{v}\rangle_{\Gamma_{N}}
$$

But, using the fact that $\mathbf{e}(\mathbf{v})=\nabla \mathbf{v}-\boldsymbol{\omega}(\mathbf{v})$, we can rewrite the first term of the latter as $(\mathbf{T}, \nabla \mathbf{v})_{\Omega}-(\mathbf{T}, \boldsymbol{\omega}(\mathbf{v}))_{\Omega}$ and then integrating by parts the term $(\mathbf{T}, \nabla \mathbf{v})_{\Omega}$, we get

$$
-(\operatorname{div} \mathbf{T}, \mathbf{v})_{\Omega}+\langle\mathbf{T n}, \mathbf{v}\rangle_{\Gamma_{N}}-\frac{1}{2}(\operatorname{as}(\mathbf{T}), \operatorname{curl}(\mathbf{v}))_{\Omega}+\langle\mathbf{u} \cdot \mathbf{n}, \mathbf{u} \cdot \mathbf{v}\rangle_{\Gamma_{N}}=(\mathbf{f}, \mathbf{v})_{\Omega}+\langle\mathbf{g}, \mathbf{v}\rangle_{\Gamma_{N}}
$$


Above we have used the identity

$$
\langle(\mathbf{u} \otimes \mathbf{w}) \mathbf{n}, \mathbf{v}\rangle_{\Gamma_{N}}=\langle\mathbf{w} \cdot \mathbf{n}, \mathbf{u} \cdot \mathbf{v}\rangle_{\Gamma_{N}} .
$$

Finally, in order to ensure the well-posedness of the resulting variational formulation, we proceed similarly as in Camaño et al. (2017), and incorporate the following redundant terms arising from the constitutive and equilibrium equations

$$
\begin{aligned}
\kappa_{1}(\operatorname{div} \mathbf{T}+\mathbf{f}, \operatorname{div} \mathbf{S})_{\Omega} & =0, \\
\kappa_{2}\left(\mathbf{T}^{\mathrm{d}}-2 v \mathbf{e}(\mathbf{u})+(\mathbf{u} \otimes \mathbf{u})^{\mathrm{d}}, \mathbf{e}(\mathbf{v})\right)_{\Omega} & =0,
\end{aligned}
$$

for all $\mathbf{S} \in \mathbb{H}(\operatorname{div} ; \Omega)$ and $\mathbf{v} \in \mathbf{H}_{\Gamma_{D}}^{1}(\Omega)$, respectively, where $\kappa_{1}$ and $\kappa_{2}$ are positive parameters to be specified later.

According to the foregoing reasoning, defining the global space

$$
\mathbf{X}:=\mathbb{H}(\mathbf{d i v} ; \Omega) \times \mathbf{H}_{\Gamma_{D}}^{1}(\Omega),
$$

endowed with the norm

$$
\|(\mathbf{S}, \mathbf{v})\|_{\mathbf{X}}^{2}:=\|\mathbf{S}\|_{\mathbf{d i v}, \Omega}^{2}+\|\mathbf{v}\|_{1, \Omega}^{2} \quad \forall(\mathbf{S}, \mathbf{v}) \in \mathbf{X}
$$

the forms

$$
\begin{aligned}
\mathbf{A}((\mathbf{T}, \mathbf{u}),(\mathbf{S}, \mathbf{v})):= & \left(\mathbf{T}^{\mathrm{d}}, \mathbf{S}^{\mathrm{d}}\right)_{\Omega}+\kappa_{1}(\operatorname{div} \mathbf{T}, \operatorname{div} \mathbf{S})_{\Omega}+2 v(\mathbf{u}, \operatorname{div} \mathbf{S})_{\Omega}-2 v(\operatorname{div} \mathbf{T}, \mathbf{v}) \\
& +v(\operatorname{curl}(\mathbf{u}), \operatorname{as}(\mathbf{S}))_{\Omega}-v(\operatorname{as}(\mathbf{T}), \operatorname{curl}(\mathbf{v}))-2 v\langle\mathbf{S n}, \mathbf{u}\rangle_{\Gamma_{N}}+2 v\langle\mathbf{T n}, \mathbf{v}\rangle_{\Gamma_{N}} \\
& +2 v \kappa_{2}(\mathbf{e}(\mathbf{u}), \mathbf{e}(\mathbf{v}))_{\Omega}-\kappa_{2}\left(\mathbf{T}^{\mathrm{d}}, \mathbf{e}(\mathbf{v})\right)_{\Omega}, \\
\mathbf{C}_{\mathbf{w}}((\mathbf{T}, \mathbf{u}),(\mathbf{S}, \mathbf{v})):= & \left((\mathbf{u} \otimes \mathbf{w})^{\mathrm{d}}, \mathbf{S}\right)_{\Omega}+2 v\langle\mathbf{w} \cdot \mathbf{n}, \mathbf{u} \cdot \mathbf{v}\rangle_{\Gamma_{N}}-\kappa_{2}\left((\mathbf{u} \otimes \mathbf{w})^{\mathrm{d}}, \mathbf{e}(\mathbf{v})\right)_{\Omega},
\end{aligned}
$$

for all $(\mathbf{T}, \mathbf{u}),(\mathbf{S}, \mathbf{v}) \in \mathbf{X}$ and $\mathbf{w} \in \mathbf{H}_{\Gamma_{D}}^{1}(\Omega)$, and the functional

$$
\mathbf{F}(\mathbf{S}, \mathbf{v}):=2 v(\mathbf{f}, \mathbf{v})_{\Omega}-\kappa_{1}(\mathbf{f}, \operatorname{div} \mathbf{S})_{\Omega}+2 v\langle\mathbf{g}, \mathbf{v}\rangle_{\Gamma_{N}} \quad \forall(\mathbf{S}, \mathbf{v}) \in \mathbf{X},
$$

multiplying (3.4) by $2 v$ and summing up the equations (3.2), 3.4, 3.6) and 3.7) we arrive at our variational problem: Given $\mathbf{f} \in \mathbf{L}^{2}(\Omega)$ and $\mathbf{g} \in \mathbf{H}_{00}^{-1 / 2}\left(\Gamma_{N}\right)$, find $(\mathbf{T}, \mathbf{u}) \in \mathbf{X}$, such that

$$
\mathbf{A}((\mathbf{T}, \mathbf{u}),(\mathbf{S}, \mathbf{v}))+\mathbf{C}_{\mathbf{u}}((\mathbf{T}, \mathbf{u}),(\mathbf{S}, \mathbf{v}))=\mathbf{F}(\mathbf{S}, \mathbf{v}) \quad \forall(\mathbf{S}, \mathbf{v}) \in \mathbf{X} .
$$

\subsection{Analysis of the continuous problem}

In what follows we prove the existence and uniqueness of solution of problem 3.10 by means of a fixed point strategy and the classical Babuška-Brezzi theory. We begin by introducing the associated fixed-point operator.

3.2.1 Fixed-point strategy. In view of the fixed-point strategy to be used in the proof of solvability for problem 3.10, let us consider [1.1] and decompose the fluid nonlinear-pseudostress as

$$
\mathbf{T}=\mathbf{T}_{0}+\mu \mathrm{I} \quad \text { with } \quad \mathbf{T}_{0} \in \mathbb{H}_{0}(\operatorname{div} ; \Omega) \quad \text { and } \quad \mu \in \mathbb{R} .
$$

By doing that, 3.4 becomes

$$
-\left(\operatorname{div} \mathbf{T}_{0}, \mathbf{v}\right)_{\Omega}+\left\langle\mathbf{T}_{0} \mathbf{n}, \mathbf{v}\right\rangle_{\Gamma_{N}}+\mu\langle\mathbf{v} \cdot \mathbf{n}, 1\rangle_{\Gamma_{N}}-\frac{1}{2}\left(\operatorname{as}\left(\mathbf{T}_{0}\right), \operatorname{curl}(\mathbf{v})\right)_{\Omega}+\langle\mathbf{u} \cdot \mathbf{n}, \mathbf{u} \cdot \mathbf{v}\rangle_{\Gamma_{N}}=(\mathbf{f}, \mathbf{v})_{\Omega}+\langle\mathbf{g}, \mathbf{v}\rangle_{\Gamma_{N}}
$$

and equation 3.2 is rewritten as

$$
\begin{array}{r}
\left(\mathbf{T}_{0}^{\mathrm{d}}, \mathbf{S}^{\mathrm{d}}\right)_{\Omega}+2 v(\mathbf{u}, \operatorname{div} \mathbf{S})_{\Omega}+v(\operatorname{curl}(\mathbf{u}), \operatorname{as}(\mathbf{S}))_{\Omega}+\left((\mathbf{u} \otimes \mathbf{u})^{\mathrm{d}}, \mathbf{S}\right)_{\Omega}-2 v\langle\mathbf{S n}, \mathbf{u}\rangle_{\Gamma_{N}}=0, \\
\eta\langle\mathbf{u} \cdot \mathbf{n}, 1\rangle_{\Gamma_{N}}=0,
\end{array}
$$

for all $(\mathbf{S}, \eta) \in \mathbb{H}_{0}(\mathbf{d i v} ; \Omega) \times \mathbb{R}$. Consequently, problem 3.10) can be rewritten equivalently as: Find $\left(\left(\mathbf{T}_{0}, \mathbf{u}\right), \mu\right) \in$ $\mathbf{X}_{0} \times \mathbb{R}$, such that

$$
\begin{array}{ll}
\mathbf{A}\left(\left(\mathbf{T}_{0}, \mathbf{u}\right),(\mathbf{S}, \mathbf{v})\right)+\mathbf{C}_{\mathbf{u}}\left(\left(\mathbf{T}_{0}, \mathbf{u}\right),(\mathbf{S}, \mathbf{v})\right)+\mathbf{B}((\mathbf{S}, \mathbf{v}), \mu) & =\mathbf{F}(\mathbf{S}, \mathbf{v}) \\
\mathbf{B}\left(\left(\mathbf{T}_{0}, \mathbf{u}\right), \eta\right) & =0
\end{array}
$$


for all $((\mathbf{S}, \mathbf{v}), \eta) \in \mathbf{X}_{0} \times \mathbb{R}$, where

and $\mathbf{B}$ is the bilinear form defined as

$$
\mathbf{X}_{0}:=\mathbb{H}_{0}(\operatorname{div} ; \Omega) \times \mathbf{H}_{\Gamma_{D}}^{1}(\Omega)
$$

$$
\mathbf{B}((\mathbf{S}, \mathbf{v}), \eta)=2 v \eta\langle\mathbf{v} \cdot \mathbf{n}, 1\rangle_{\Gamma_{N}} \quad \forall((\mathbf{S}, \mathbf{v}), \eta) \in \mathbf{X} \times \mathbb{R} .
$$

To be more precise, since (1.1) is an orthogonal decomposition, $\left(\left(\mathbf{T}_{0}, \mathbf{u}\right), \mu\right) \in \mathbf{X}_{0} \times \mathbb{R}$ is a solution to 3.12), if and only if, $(\mathbf{T}, \mathbf{u})=\left(\mathbf{T}_{0}+\mu \mathrm{I}, \mathbf{u}\right) \in \mathbf{X} \times \mathbb{R}$ is a solution to 3.10).

Let us now introduce the bounded set

$$
\mathbf{K}:=\left\{\mathbf{v} \in \mathbf{H}_{\Gamma_{D}}^{1}(\Omega):\|\mathbf{v}\|_{1, \Omega} \leqslant \frac{2\left\|\left.\mathbf{F}\right|_{\mathbf{x}_{0}}\right\|_{\mathbf{X}_{0}^{\prime}}}{\alpha_{\mathbf{A}}}\right\},
$$

and the mapping

$$
\mathscr{J}: \mathbf{K} \rightarrow \mathbf{K}, \quad \mathbf{w} \rightarrow \mathscr{J}(\mathbf{w})=\mathbf{u}
$$

with $\mathbf{u}$ being the second component of $\left(\left(\mathbf{T}_{0}, \mathbf{u}\right), \mu\right) \in \mathbf{X}_{0} \times \mathbb{R}$, solution to the linearized version of problem 3.12:

$$
\begin{array}{ll}
\mathbf{A}\left(\left(\mathbf{T}_{0}, \mathbf{u}\right),(\mathbf{S}, \mathbf{v})\right)+\mathbf{C}_{\mathbf{w}}\left(\left(\mathbf{T}_{0}, \mathbf{u}\right),(\mathbf{S}, \mathbf{v})\right)+\mathbf{B}((\mathbf{S}, \mathbf{v}), \mu) & =\mathbf{F}(\mathbf{S}, \mathbf{v}), \\
\mathbf{B}\left(\left(\mathbf{T}_{0}, \mathbf{u}\right), \eta\right) & =0,
\end{array}
$$

for all $((\mathbf{S}, \mathbf{v}), \eta) \in \mathbf{X}_{0} \times \mathbb{R}$. It is easy to see that $\left(\left(\mathbf{T}_{0}, \mathbf{u}\right), \mu\right) \in \mathbf{X}_{0} \times \mathbb{R}$ is a solution to 3.12 , if and only if, $\mathscr{J}(\mathbf{u})=\mathbf{u}$. This step, together with the equivalence between 3.10 and 3.12 , imply the following relations:

$$
\mathscr{J}(\mathbf{u})=\mathbf{u} \Leftrightarrow\left(\left(\mathbf{T}_{0}, \mathbf{u}\right), \mu\right) \in \mathbf{X}_{0} \times \mathbb{R} \text { satisfies 3.12 } \Leftrightarrow\left(\mathbf{T}_{0}+\mu \mathrm{I}, \mathbf{u}\right) \in \mathbf{X} \text { satisfies 3.10. }
$$

In this way, in establishing the well-posedness of (3.10), or equivalently 3.12 , it suffices to prove that $\mathscr{J}$ has a unique fixed point. Before proceeding with the solvability analysis, we first state the stability of the involved forms and the well-definiteness of the fixed-point operator $\mathscr{J}$.

3.2.2 Stability results and well-definiteness of $\mathscr{J}$. In this section we establish adequate stability properties, and verify the well-definiteness of $\mathscr{J}$. Since this operator is defined through a saddle-point problem, we proceed differently than in Camaño et al. (2017), and apply the Babuška-Brezzi theory.

In the sequel we make use of the following well known estimates:

$$
\begin{aligned}
& C_{d}\|\mathbf{S}\|_{0, \Omega}^{2} \leqslant\left\|\mathbf{S}^{\mathrm{d}}\right\|_{0, \Omega}^{2}+\|\operatorname{div} \mathbf{S}\|_{0, \Omega}^{2} \quad \forall \mathbf{S} \in \mathbb{H}_{0}(\operatorname{div} ; \Omega), \\
& C_{K}\|\mathbf{v}\|_{1, \Omega}^{2} \leqslant\|\mathbf{e}(\mathbf{v})\|_{0, \Omega}^{2} \leqslant\|\mathbf{v}\|_{1, \Omega}^{2} \quad \forall \mathbf{v} \in \mathbf{H}^{1}(\Omega),
\end{aligned}
$$

with $C_{d}$ and $C_{K}$ only depending on $\Omega$ (for (3.18) we refer to Lemma 3.1 in Arnold et al. 1984a or Chapter IV in Brezzi \& Fortin 1991, whereas for 3.19) we refer to Girault \& Raviart 1986).

Let us start the discussion by deriving the continuity of the forms involved. First, employing the right-hand side of 3.19, the continuity of the normal trace and simple computations it can be deduced that

$$
|\mathbf{A}((\mathbf{T}, \mathbf{u}),(\mathbf{S}, \mathbf{v}))| \leqslant C_{\mathbf{A}}\|(\mathbf{T}, \mathbf{u})\|_{\mathbf{X}}\|(\mathbf{S}, \mathbf{v})\|_{\mathbf{X}}, \quad \forall(\mathbf{T}, \mathbf{u}),(\mathbf{S}, \mathbf{v}) \in \mathbf{X},
$$

with $C_{\mathbf{A}}:=4 \max \left\{1, \kappa_{1}, 2 v, 2 v \kappa_{2}, \kappa_{2}\right\}$. In turn, employing the continuity of the embedding $\mathbf{i}_{\Gamma}: \mathbf{H}^{1 / 2}(\Gamma) \rightarrow$ $\mathbf{L}^{4}(\Gamma)$, and the continuity of the trace operator $\gamma_{0}: \mathbf{H}^{1}(\Omega) \rightarrow \mathbf{L}^{2}(\Gamma)$, it can be easily proved that

$$
\left|\langle\mathbf{w} \cdot \mathbf{n}, \mathbf{u}, \mathbf{v}\rangle_{\Gamma_{N}}\right| \leqslant\left\|\mathbf{i}_{\Gamma}\right\|^{2}\left\|\gamma_{0}\right\|\|\mathbf{w}\|_{1, \Omega}\|\mathbf{u}\|_{1 / 2, \Gamma}\|\mathbf{v}\|_{1 / 2, \Gamma}
$$

This inequality, together with 3.19) and the continuity of the embedding $\mathbf{i}: \mathbf{H}^{1}(\Omega) \rightarrow \mathbf{L}^{4}(\Omega)$ implies that

$$
\left|\mathbf{C}_{\mathbf{w}}((\mathbf{T}, \mathbf{u}),(\mathbf{S}, \mathbf{v}))\right| \leqslant C_{\mathbf{C}}\|\mathbf{w}\|_{1, \Omega}\|(\mathbf{T}, \mathbf{u})\|_{\mathbf{X}}\|(\mathbf{S}, \mathbf{v})\|_{\mathbf{X}},
$$

with $C_{\mathbf{C}}:=\|\mathbf{i}\|^{2}\left(1+\kappa_{2}^{2}\right)^{1 / 2}+2 v\left\|\mathbf{i}_{\Gamma}\right\|^{2}\left\|\gamma_{0}\right\|$. Let us now observe also that $\mathbf{F} \mid \mathbf{X}_{0} \in \mathbf{X}_{0}^{\prime}$ and

$$
\left\|\left.\mathbf{F}\right|_{\mathbf{x}_{0}}\right\| \mathbf{X}_{0}^{\prime} \leqslant\left(4 v^{2}+\kappa_{1}^{2}\right)^{1 / 2}\|\mathbf{f}\|_{0, \Omega}+2 v\|\mathbf{g}\|_{-1 / 2,00, \Gamma_{N}} .
$$

We continue the discussion by establishing the ellipticity of the form $\mathbf{A}$ on $\mathbf{X}_{0}$.

LEMmA 3.1 Assume that $\kappa_{1}>0$ and $0<\kappa_{2}<4 v$. Then there exists $\alpha_{\mathrm{A}}>0$, such that

$$
\mathbf{A}((\mathbf{S}, \mathbf{v}),(\mathbf{S}, \mathbf{v})) \geqslant \alpha_{\mathbf{A}}\|(\mathbf{S}, \mathbf{v})\|_{\mathbf{X}}^{2} \quad \forall(\mathbf{S}, \mathbf{v}) \in \mathbf{X}_{0} .
$$


Proof. From the definition of A (cf. [3.8), we have

$$
\mathbf{A}((\mathbf{S}, \mathbf{v}),(\mathbf{S}, \mathbf{v}))=\left\|\mathbf{S}^{\mathrm{d}}\right\|_{0, \Omega}^{2}+\kappa_{1}\|\operatorname{div} \mathbf{S}\|_{0, \Omega}^{2}+2 \kappa_{2} v\|\mathbf{e}(\mathbf{v})\|_{0, \Omega}^{2}-\kappa_{2}\left(\mathbf{S}^{\mathrm{d}}, \mathbf{e}(\mathbf{v})\right)_{\Omega} .
$$

Then, we proceed analogously to the proof of Lemma 3.1 in Camaño et al. (2017), and apply inequalities (3.18) and 3.19, to obtain 3.23, with $\alpha_{\mathbf{A}}:=\frac{1}{2} \min \left\{\min \left\{1, \kappa_{1}\right\}, \kappa_{1}, \kappa_{2}\left(4 v-\kappa_{2}\right) C_{K}\right\}$.

Finally, we establish the inf-sup condition of the bilinear form $\mathbf{B}$.

LEMmA 3.2 There exists $\beta>0$, such that

$$
\sup _{(\mathbf{S}, \mathbf{v}) \in \mathbf{X}_{0} \backslash\{\mathbf{0}\}} \frac{\mathbf{B}((\mathbf{S}, \mathbf{v}), \eta)}{\|(\mathbf{S}, \mathbf{v})\|_{\mathbf{X}}} \geqslant \beta|\eta| \quad \forall \eta \in \mathbb{R} .
$$

Proof. Let $\mathbf{v}_{0} \in \mathbf{H}_{\Gamma_{D}}^{1}(\Omega)$ be a fixed element such that $\left\langle\mathbf{v}_{0} \cdot \mathbf{n}, 1\right\rangle_{\Gamma_{N}} \neq 0$. Hence, given $\eta \in \mathbb{R}$, it follows that

$$
\sup _{(\mathbf{S}, \mathbf{v}) \in \mathbf{X}_{0} \backslash\{\mathbf{0}\}} \frac{\mathbf{B}((\mathbf{S}, \mathbf{v}), \eta)}{\|(\mathbf{S}, \mathbf{v})\|_{\mathbf{X}}} \geqslant \frac{\left|\mathbf{B}\left(\left(\mathbf{0}, \mathbf{v}_{0}\right), \eta\right)\right|}{\left\|\left(\mathbf{0}, \mathbf{v}_{0}\right)\right\|_{\mathbf{X}}}=\frac{2 v\left\langle\mathbf{v}_{0} \cdot \mathbf{n}, 1\right\rangle}{\left\|\mathbf{v}_{0}\right\|_{1, \Omega}}|\eta|,
$$

which implies the result with $\beta=\frac{2 v\left\langle\mathbf{v}_{0} \cdot \mathbf{n}, 1\right\rangle}{\left\|\mathbf{v}_{0}\right\|_{1, \Omega}}$.

Note that there is a very simple way of defining such an element $\mathbf{v}_{0}$. In fact, for the two dimensional case, if $x_{1}$ and $x_{2}$ are two points on $\Gamma$ such that the line $\left[x_{1}, x_{2}\right] \subseteq \Gamma_{N}$, then we let $x_{m}$ be the midpoint of $\left[x_{1}, x_{2}\right]$ and $x_{3}$ be a point on $\Gamma$, or in the interior of $\Omega$, in such a way two triangles $T_{1}$ and $T_{2}$ can be constructed (see the left panel in Figure 3.1. Then, we let $v \in \mathrm{H}_{\Gamma_{N}}^{1}(\Omega)$ be such that $\left.v\right|_{T_{i}} \in P_{1}\left(T_{i}\right)$, for $i=1,2, v\left(x_{m}\right)=1, v\left(x_{i}\right)=0$ for $i=1,2,3$, and $v(x)=0$ for all $x \in \bar{\Omega}-\left\{T_{1} \cup T_{2}\right\}$, and define $\mathbf{v}_{0}=v \mathbf{n}$, where $\mathbf{n}$ is the normal vector on $\left[x_{1}, x_{2}\right]$ (see Gatica et al. 2011b Lemma 3.6 for a similar approach). Similarly, for the three dimensional case, given $x_{1}, x_{2}, x_{3}$ three points on $\Gamma_{N}$ defining a triangle with barycentric $x_{m}$ (see the right-hand side of Figure 3.1 and $x_{4}$ a point in the interior of $\Omega$, or on an opposite piece of boundary, chosen in such a way below $x_{4}$ can be formed three tetrahedra $T_{1}, T_{2}, T_{3}$. Then we let $v \in \mathrm{H}_{\Gamma_{N}}^{1}(\Omega)$ be such that $\left.v\right|_{T_{i}} \in P_{1}\left(T_{i}\right)$, for $i=1,2,3, v\left(x_{m}\right)=1$, $v\left(x_{i}\right)=0$ for $i=1,2,3,4$, and $v(x)=0$ for all $x \in \bar{\Omega}-\left\{T_{1} \cup T_{2} \cup T_{3}\right\}$, and define $\mathbf{v}_{0}=v \mathbf{n}$, where $\mathbf{n}$ is the normal vector on $T_{1} \cup T_{2} \cup T_{3}$.
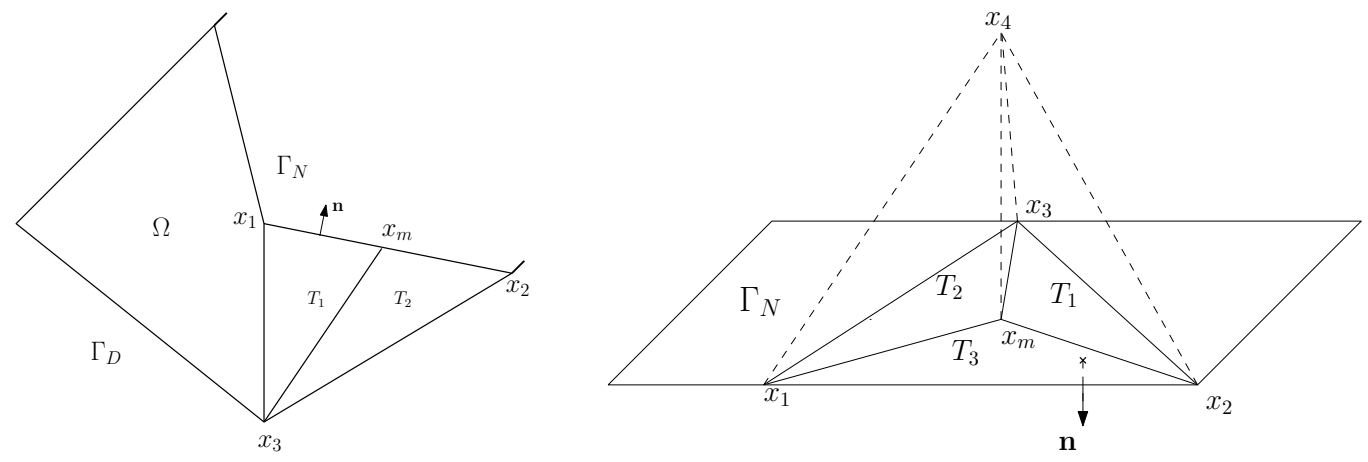

FIG. 3.1. Choice of $\mathbf{v}_{0}$ for the two (left) and three (right) dimensional cases.

We are now in position of establishing the well-definiteness of operator $\mathscr{J}$.

Lemma 3.3 Assume that $\kappa_{1}>0$ and $0<\kappa_{2}<4 v$. Assume further that the external forces $\mathbf{f}$ and $\mathbf{g}$ satisfy

$$
\left(4 v^{2}+\kappa_{1}^{2}\right)^{1 / 2}\|\mathbf{f}\|_{0, \Omega}+2 v\|\mathbf{g}\|_{-1 / 2,00, \Gamma_{N}} \leqslant \frac{\alpha_{\mathbf{A}}^{2}}{4 C_{\mathbf{C}}}
$$

with $\alpha_{\mathbf{A}}$ and $C_{\mathbf{C}}$ being the constants in 3.23 and 3.21, respectively. Then, given $\mathbf{w} \in \mathbf{K}$, there exists a unique $\mathbf{u} \in \mathbf{K}$, such that $\mathscr{J}(\mathbf{w})=\mathbf{u}$.

Proof. Let $\mathbf{w} \in \mathbf{K}$. Notice first that, owing to 3.22, 3.25] and the definition of $\mathbf{K}$, it readily follows that

$$
\|\mathbf{w}\|_{1, \Omega} \leqslant \frac{\alpha_{\mathbf{A}}}{2 C_{\mathbf{C}}} .
$$


In turn, from 3.21 and 3.23, there holds

$$
\begin{aligned}
\mathbf{A}((\mathbf{S}, \mathbf{v}),(\mathbf{S}, \mathbf{v}))+\mathbf{C}_{\mathbf{w}}((\mathbf{S}, \mathbf{v}),(\mathbf{S}, \mathbf{v})) & \geqslant \mathbf{A}((\mathbf{S}, \mathbf{v}),(\mathbf{S}, \mathbf{v}))-\left|\mathbf{C}_{\mathbf{w}}((\mathbf{S}, \mathbf{v}),(\mathbf{S}, \mathbf{v}))\right|, \\
& \geqslant\left(\alpha_{\mathbf{A}}-C_{\mathbf{C}}\|\mathbf{w}\|_{1, \Omega}\right)\|(\mathbf{S}, \mathbf{v})\|_{\mathbf{X}}^{2} \quad \forall(\mathbf{S}, \mathbf{v}) \in \mathbf{X}_{0},
\end{aligned}
$$

which together with 3.26, implies

$$
\mathbf{A}((\mathbf{S}, \mathbf{v}),(\mathbf{S}, \mathbf{v}))+\mathbf{C}_{\mathbf{w}}((\mathbf{S}, \mathbf{v}),(\mathbf{S}, \mathbf{v})) \geqslant \frac{\alpha_{\mathbf{A}}}{2}\|(\mathbf{S}, \mathbf{v})\|_{\mathbf{X}}^{2} \quad(\mathbf{S}, \mathbf{v}) \in \mathbf{X}_{0}
$$

that is, the bilinear form $\mathbf{A}(\cdot, \cdot)+\mathbf{C}_{\mathbf{w}}(\cdot, \cdot)$ is elliptic on $\mathbf{X}_{0}$. Therefore, since the bilinear form $\mathbf{B}$ satisfies (3.24), we can apply the classical Babuška-Brezzi theory to deduce that there exists a unique $\left(\left(\mathbf{T}_{0}, \mathbf{u}\right), \mu\right) \in \mathbf{X}_{0} \times \mathbb{R}$ satisfying 3.16. Moreover, since $\mathbf{B}\left(\left(\mathbf{T}_{0}, \mathbf{u}\right), \mu\right)=0$, from the first equation of 3.16 and from 3.28) we easily obtain that

$$
\frac{\alpha_{\mathbf{A}}}{2}\left\|\left(\mathbf{T}_{0}, \mathbf{u}\right)\right\|_{\mathbf{X}}^{2} \leqslant \mathbf{A}\left(\left(\mathbf{T}_{0}, \mathbf{u}\right),\left(\mathbf{T}_{0}, \mathbf{u}\right)\right)+\mathbf{C}_{\mathbf{w}}\left(\left(\mathbf{T}_{0}, \mathbf{u}\right),\left(\mathbf{T}_{0}, \mathbf{u}\right)\right)=\mathbf{F}\left(\mathbf{T}_{0}, \mathbf{u}\right),
$$

which implies that

$$
\|\mathbf{u}\|_{1, \Omega} \leqslant\left\|\left(\mathbf{T}_{0}, \mathbf{u}\right)\right\|_{\mathbf{X}} \leqslant \frac{2}{\alpha_{\mathbf{A}}}\left\|\left.\mathbf{F}\right|_{\mathbf{X}_{0}}\right\|_{\mathbf{X}_{0}^{\prime}}
$$

and hence $\mathbf{u} \in \mathbf{K}$.

3.2.3 The main result. The main result of this section states the unique solvability and stability estimates for problem 3.10.

THEOREM 3.1 Let $\mathbf{f} \in \mathbf{L}^{2}(\Omega)$ and $\mathbf{g} \in \mathbf{H}_{00}^{-1 / 2}\left(\Gamma_{N}\right)$, such that

$$
\left(4 v^{2}+\kappa_{1}^{2}\right)^{1 / 2}\|\mathbf{f}\|_{0, \Omega}+2 v\|\mathbf{g}\|_{-1 / 2,00, \Gamma_{N}}<\frac{\alpha_{\mathbf{A}}^{2}}{4 C_{\mathbf{C}}} .
$$

Assume that $\kappa_{1}>0$ and $0<\kappa_{2}<4 v$. Then, there exists a unique $(\mathbf{T}, \mathbf{u}) \in \mathbf{X}$ solution to [3.10]. In addition, the solution $(\mathbf{T}, \mathbf{u})$ satisfies the estimate

$$
\|(\mathbf{T}, \mathbf{u})\|_{\mathbf{X}} \leqslant 2\left(\frac{1}{\alpha_{\mathbf{A}}}+n^{1 / 2}|\Omega|^{1 / 2}\left(\frac{1}{\beta}+\frac{C_{\mathbf{A}}}{\beta \alpha_{\mathbf{A}}}\right)\right)\left(\left(4 v^{2}+\kappa_{1}^{2}\right)^{1 / 2}\|\mathbf{f}\|_{0, \Omega}+2 v\|\mathbf{g}\|_{-1 / 2,00, \Gamma_{N}}\right) .
$$

Proof. First, let us observe that assumption 3.30) ensures that Lemma 3.3 holds true (see assumption 3.25). To prove the well-posedness of (3.10), in what follows we make use of the classical Banach's fixed point Theorem and prove equivalently that the mapping $\mathscr{J}$ has a unique fixed point in $\mathbf{K}$. To do that we let $\mathbf{z}_{1}, \mathbf{z}_{2}$ and $\mathbf{u}_{1}, \mathbf{u}_{2} \in \mathbf{K}$, such that $\mathbf{u}_{1}=\mathscr{J}\left(\mathbf{z}_{1}\right)$ and $\mathbf{u}_{2}=\mathscr{J}\left(\mathbf{z}_{2}\right)$. From the definition of $\mathscr{J}$ it follows that

$$
\begin{aligned}
\mathbf{A}\left(\left(\mathbf{T}_{i}, \mathbf{u}_{i}\right),(\mathbf{S}, \mathbf{v})\right)+\mathbf{C}_{\mathbf{z}_{i}}\left(\left(\mathbf{T}_{i}, \mathbf{u}_{i}\right),(\mathbf{S}, \mathbf{v})\right)+\mathbf{B}\left((\mathbf{S}, \mathbf{v}), \mu_{i}\right) & =\mathbf{F}(\mathbf{S}, \mathbf{v}) & & \forall(\mathbf{S}, \mathbf{v}) \in \mathbf{X}_{0}, \\
\mathbf{B}\left(\left(\mathbf{T}_{i}, \mathbf{u}_{i}\right), \eta\right) & =0 & & \forall \eta \in \mathbb{R},
\end{aligned}
$$

for $i=1,2$, where $\mathbf{T}_{i} \in \mathbb{H}_{0}(\operatorname{div} ; \Omega)$ and $\mu_{i} \in \mathbb{R}$ are the elements that together with $\mathbf{u}_{i}$ constitute the unique solution of (3.16) with $\mathbf{z}_{i}$. Then, by subtracting equations (3.32) with $i=1,2$, choosing the test function $((\mathbf{S}, \mathbf{v}), \eta)=\left(\left(\mathbf{T}_{1}-\mathbf{T}_{2}, \mathbf{u}_{1}-\mathbf{u}_{2}\right), \mu_{1}-\mu_{2}\right) \in \mathbf{X}_{0} \times \mathbb{R}$, applying the ellipticity of $\mathbf{A}(\cdot, \cdot)+\mathbf{C}_{\mathbf{z}_{2}}(\cdot, \cdot)$ on $\mathbf{X}_{0}$ (cf. (3.28), the continuity of $\mathbf{C}_{\mathbf{z}_{1}-\mathbf{z}_{2}}$ (cf. (3.21), and the fact that $\left\|\left(\mathbf{T}_{1}, \mathbf{u}_{1}\right)\right\| \mathbf{X} \leqslant \frac{2}{\alpha_{A}}\left\|\left.\mathbf{F}\right|_{\mathbf{x}_{0}}\right\|_{\mathbf{X}_{0}^{\prime}}$ (cf. 3.29), we proceed similarly as in the proof of Theorem 3.4 in Camaño et al. (2017) to obtain

$$
\left\|\mathbf{u}_{1}-\mathbf{u}_{2}\right\|_{1, \Omega} \leqslant\left\|\left(\mathbf{T}_{1}-\mathbf{T}_{2}, \mathbf{u}_{1}-\mathbf{u}_{2}\right)\right\| \mathbf{x} \leqslant \frac{2 C_{\mathbf{C}}}{\alpha_{\mathbf{A}}}\left\|\left(\mathbf{T}_{1}, \mathbf{u}_{1}\right)\right\| \mathbf{x}\left\|\mathbf{z}_{1}-\mathbf{z}_{2}\right\|_{1, \Omega} \leqslant \frac{4 C_{\mathbf{C}}\left\|\left.\mathbf{F}\right|_{\mathbf{x}_{0}}\right\| \mathbf{x}_{0}^{\prime}}{\alpha_{\mathbf{A}}^{2}}\left\|\mathbf{z}_{1}-\mathbf{z}_{2}\right\|_{1, \Omega} .
$$

Hence, employing $(3.22$ and assumption $(3.30)$, from $(3.33)$ we readily obtain that $\mathscr{J}$ is a contraction mapping. In this way, by applying the Banach's fixed point Theorem we obtain that there exists a unique $\mathbf{u} \in \mathbf{K}$ such that $\mathscr{J}(\mathbf{u})=\mathbf{u}$, or equivalently, there exists a unique $(\mathbf{T}, \mathbf{u}) \in \mathbf{X}$ solution to 3.10 .

Now, to deduce estimate 3.31 we consider the decomposition $\mathbf{T}=\mathbf{T}_{0}+\mu \mathrm{I}$ and recall that $\left(\left(\mathbf{T}_{0}, \mathbf{u}\right), \mu\right) \in$ $\mathbf{X}_{0} \times \mathbb{R}$ is the unique solution of $(3.12)$ (see (3.17)). Then, we first utilize the fact that $\mathbf{A}(\cdot, \cdot)+\mathbf{C}_{\mathbf{u}}(\cdot, \cdot)$ is elliptic on $\mathbf{X}_{0}$, and proceed analogously as in the proof of Lemma 3.3 (see 3.29), to obtain

$$
\left\|\left(\mathbf{T}_{0}, \mathbf{u}\right)\right\|_{\mathbf{X}} \leqslant \frac{2}{\alpha_{\mathbf{A}}}\left\|\left.\mathbf{F}\right|_{\mathbf{X}_{0}}\right\|_{\mathbf{X}_{0}^{\prime}} .
$$


Moreover, using 3.22, 3.30, from 3.34, we also obtain

$$
\|\mathbf{u}\|_{1, \Omega} \leqslant\left\|\left(\mathbf{T}_{0}, \mathbf{u}\right)\right\| \mathbf{x} \leqslant \frac{\alpha_{\mathbf{A}}}{2 C_{\mathbf{C}}} .
$$

In turn, utilizing the inf-sup condition 3.24 , the first equation of 3.12 , and the continuity of $\mathbf{F}, \mathbf{A}$ and $\mathbf{C}_{\mathbf{u}}$, we get

$$
\begin{aligned}
\beta|\mu| & \leqslant \sup _{(\mathbf{S}, \mathbf{v}) \in \mathbf{X}_{0} \backslash\{\mathbf{0}\}} \frac{\mathbf{B}((\mathbf{S}, \mathbf{v}), \mu)}{\|(\mathbf{S}, \mathbf{v})\|_{\mathbf{X}}} \\
& =\sup _{(\mathbf{S}, \mathbf{v}) \in \mathbf{X}_{0} \backslash\{\mathbf{0}\}} \frac{\mathbf{F}(\mathbf{S}, \mathbf{v})-\mathbf{A}\left(\left(\mathbf{T}_{0}, \mathbf{u}\right),(\mathbf{S}, \mathbf{v})\right)-\mathbf{C}_{\mathbf{u}}\left(\left(\mathbf{T}_{0}, \mathbf{u}\right),(\mathbf{S}, \mathbf{v})\right)}{\|(\mathbf{S}, \mathbf{v})\|_{\mathbf{X}}} \\
& \leqslant\left\|\left.\mathbf{F}\right|_{\mathbf{X}_{0}}\right\|_{\mathbf{X}_{0}^{\prime}}+C_{\mathbf{A}}\left\|\left(\mathbf{T}_{0}, \mathbf{u}\right)\right\|_{\mathbf{X}}+C_{\mathbf{C}}\|\mathbf{u}\|_{1, \Omega}\left\|\left(\mathbf{T}_{0}, \mathbf{u}\right)\right\|_{\mathbf{X}},
\end{aligned}
$$

which combined with 3.34 and 3.35 yields

$$
|\mu| \leqslant \frac{1}{\beta}\left(1+\frac{2 C_{\mathbf{A}}}{\alpha_{\mathbf{A}}}+\frac{2 C_{\mathbf{C}}\|\mathbf{u}\|_{1, \Omega}}{\alpha_{\mathbf{A}}}\right)\left\|\left.\mathbf{F}\right|_{\mathbf{X}_{0}}\right\|_{\mathbf{X}_{0}^{\prime}} \leqslant \frac{2}{\beta}\left(1+\frac{C_{\mathbf{A}}}{\alpha_{\mathbf{A}}}\right)\left\|\left.\mathbf{F}\right|_{\mathbf{X}_{0}}\right\|_{\mathbf{X}_{0}^{\prime}}
$$

Therefore, recalling that $\|\mathrm{I}\|_{\operatorname{div}, \Omega}^{2}=(n, 1)_{\Omega}=n|\Omega|$, from 3.34) and 3.37) we easily see that

$$
\|(\mathbf{T}, \mathbf{u})\|_{\mathbf{X}} \leqslant\left\|\left(\mathbf{T}_{0}, \mathbf{u}\right)\right\|_{\mathbf{X}}+\|(\mu \mathrm{I}, \mathbf{0})\|_{\mathbf{X}} \leqslant 2\left(\frac{1}{\alpha_{\mathbf{A}}}+n^{1 / 2}|\Omega|^{1 / 2}\left(\frac{1}{\beta}+\frac{C_{\mathbf{A}}}{\beta \alpha_{\mathbf{A}}}\right)\right)\left\|\left.\mathbf{F}\right|_{\mathbf{X}_{0}}\right\|_{\mathbf{X}_{0}^{\prime}},
$$

which together with 3.22 implies 3.31.

\section{The Galerkin scheme}

Most results in this section are simply inherited (or can be readily deduced) from their continuous counterpart. Details will be therefore omitted whenever appropriate.

\subsection{Discrete problem}

We start by introducing the generic finite dimensional subspaces

$$
\mathbf{H}_{h}(\operatorname{div} ; \Omega) \subseteq \mathbf{H}(\operatorname{div} ; \Omega), \quad \mathrm{H}_{h, D}^{1}(\Omega) \subseteq \mathrm{H}_{\Gamma_{D}}^{1}(\Omega) .
$$

Then defining

$$
\begin{aligned}
\mathbb{H}_{h} & :=\left\{\mathbf{S}_{h} \in \mathbb{H}(\mathbf{d i v} ; \Omega): \mathbf{c}^{t} \mathbf{S}_{h} \in \mathbf{H}_{h}(\operatorname{div} ; \Omega) \quad \forall \mathbf{c} \in \mathbb{R}^{n}\right\} \subseteq \mathbb{H}(\operatorname{div} ; \Omega), \\
\mathbf{H}_{h, D}^{1} & :=\left[\mathrm{H}_{h, D}^{1}(\Omega)\right]^{n} \subseteq \mathbf{H}_{\Gamma_{D}}^{1}(\Omega), \\
\mathbf{X}_{h} & :=\mathbb{H}_{h} \times \mathbf{H}_{h, D}^{1} \subseteq \mathbf{X},
\end{aligned}
$$

our Galerkin scheme reads: Find $\left(\mathbf{T}_{h}, \mathbf{u}_{h}\right) \in \mathbf{X}_{h}$, such that

$$
\mathbf{A}\left(\left(\mathbf{T}_{h}, \mathbf{u}_{h}\right),\left(\mathbf{S}_{h}, \mathbf{v}_{h}\right)\right)+\mathbf{C}_{\mathbf{u}_{h}}\left(\left(\mathbf{T}_{h}, \mathbf{u}_{h}\right),\left(\mathbf{S}_{h}, \mathbf{v}_{h}\right)\right)=\mathbf{F}\left(\mathbf{S}_{h}, \mathbf{v}_{h}\right) \quad \forall\left(\mathbf{S}_{h}, \mathbf{v}_{h}\right) \in \mathbf{X}_{h},
$$

with $\mathbf{A}, \mathbf{C}$ and $\mathbf{F}$ defined in 3.8 and 3.9 .

In the subsequent sections we prove that under suitable assumptions on the finite dimensional spaces $\mathbf{H}_{h}(\operatorname{div} ; \Omega)$ and $\mathrm{H}_{h, D}^{1}(\Omega)$, problem 4.3 is well posed. Our approach basically consists of adapting to the present discrete case the arguments employed in the analysis of the continuous problem, mainly those from the proofs of Lemmas 3.1, 3.2 and 3.3 and Theorem 3.1

We begin the derivation of the aforementioned assumptions by observing that, in order to perform the decomposition 1.1 we need to eliminate multiples of the identity matrix from $\mathbb{H}_{h}$. This request is certainly satisfied if we assume that:

$$
\mathbf{P}_{0}(\Omega) \subseteq \mathbf{H}_{h}(\mathbf{d i v} ; \Omega) .
$$

In particular, it follows that $\mathrm{I} \in \mathbb{H}_{h}$ for all $h$, and hence there holds the decomposition:

$$
\mathbb{H}_{h}=\mathbb{H}_{h, 0} \oplus P_{0}(\Omega) \mathrm{I} \quad \text { with } \quad \mathbb{H}_{h, 0}:=\mathbb{H}_{h} \cap \mathbb{H}_{0}(\operatorname{div} ; \Omega),
$$


and, analogously to the continuous case, problem (4.3) can be rewritten equivalently as: Find $\left(\left(\mathbf{T}_{h, 0}, \mathbf{u}_{h}\right), \mu_{h}\right) \in \mathbf{X}_{h, 0} \times \mathbb{R}$, such that

$$
\begin{array}{ll}
\mathbf{A}\left(\left(\mathbf{T}_{h, 0}, \mathbf{u}_{h}\right),\left(\mathbf{S}_{h}, \mathbf{v}_{h}\right)\right)+\mathbf{C}_{\mathbf{u}_{h}}\left(\left(\mathbf{T}_{h, 0}, \mathbf{u}_{h}\right),\left(\mathbf{S}_{h}, \mathbf{v}_{h}\right)\right)+\mathbf{B}\left(\left(\mathbf{S}_{h}, \mathbf{v}_{h}\right), \mu_{h}\right) & =\mathbf{F}\left(\mathbf{S}_{h}, \mathbf{v}_{h}\right), \\
\mathbf{B}\left(\left(\mathbf{T}_{h, 0}, \mathbf{u}_{h}\right), \eta_{h}\right) & =0,
\end{array}
$$

for all $\left(\left(\mathbf{S}_{h}, \mathbf{v}_{h}\right), \eta_{h}\right) \in \mathbf{X}_{h, 0} \times \mathbb{R}$, where

$$
\mathbf{X}_{h, 0}:=\mathbb{H}_{h, 0} \times \mathbf{H}_{h, D}^{1},
$$

and $\mathbf{B}$ is the bilinear form defined in 3.13).

Next, following the same arguments utilized in the proof of Lemma 3.2 we deduce that $\mathbf{B}$ satisfies the discrete version of 3.24, namely,

$$
\sup _{\left(\mathbf{S}_{h}, \mathbf{v}_{h}\right) \in \mathbf{X}_{h, 0} \backslash\{\mathbf{0}\}} \frac{\mathbf{B}\left(\left(\mathbf{S}_{h}, \mathbf{v}_{h}\right), \eta_{h}\right)}{\left\|\left(\mathbf{S}_{h}, \mathbf{v}_{h}\right)\right\|_{\mathbf{X}}} \geqslant \tilde{\beta}\left|\eta_{h}\right| \quad \forall \eta_{h} \in \mathbb{R},
$$

with $\tilde{\beta}>0$, independent of the discretization parameter $h$, if and only if, there holds:

(H.1) There exists $\hat{\mathbf{v}}_{0} \in \mathbf{H}_{\Gamma_{D}}^{1}(\Omega)$, such that

$$
\hat{\mathbf{v}}_{0} \in \mathbf{H}_{h, D}^{1} \quad \forall h>0 \quad \text { and } \quad\left\langle\hat{\mathbf{v}}_{0} \cdot \mathbf{n}, 1\right\rangle_{\Gamma_{N}} \neq 0 .
$$

In fact, utilizing (H.1), it is clear that

$$
\sup _{\left(\mathbf{S}_{h}, \mathbf{v}_{h}\right) \in \mathbf{X}_{h, 0} \backslash\{\mathbf{0}\}} \frac{\mathbf{B}\left(\left(\mathbf{S}_{h}, \mathbf{v}_{h}\right), \eta_{h}\right)}{\left\|\left(\mathbf{S}_{h}, \mathbf{v}_{h}\right)\right\|_{\mathbf{X}}} \geqslant \frac{\mathbf{B}\left(\left(\mathbf{0}, \hat{\mathbf{v}}_{0}\right), \eta_{h}\right)}{\left\|\hat{\mathbf{v}}_{0}\right\|_{1, \Omega}}=\tilde{\beta}\left|\eta_{h}\right|,
$$

with $\tilde{\beta}=\frac{\left\langle\hat{\mathbf{v}}_{0} \cdot \mathbf{n}, 1\right\rangle}{\left\|\hat{\mathbf{v}}_{0}\right\|_{1, \Omega}}$ independent of $h$.

We now adapt the fixed-point strategy from the continuous case to prove the well-posedness of 4.3 .

\subsection{Fixed-point strategy and well-posedness analysis}

We begin by observing that, since $\mathbb{H}_{h}$ and $\mathbf{H}_{h, D}^{1}$ are subspaces of $\mathbb{H}(\mathbf{d i v} ; \Omega)$ and $\mathbf{H}_{\Gamma_{D}}^{1}(\Omega)$, respectively, the estimates (3.20), (3.21) and (3.22) are inherited, as well as the ellipticity of $\mathbf{A}$ on $\mathbf{X}_{h, 0}$.

Analogously to the continuous case, let us introduce the finite dimensional bounded set

$$
\mathbf{K}_{h}:=\left\{\mathbf{v}_{h} \in \mathbf{H}_{h, D}^{1}:\left\|\mathbf{v}_{h}\right\|_{1, \Omega} \leqslant \frac{2\left\|\mathbf{F} \mid \mathbf{x}_{h, 0}\right\|_{\mathbf{X}_{h, 0}^{\prime}}}{\alpha_{\mathbf{A}}}\right\},
$$

where $\alpha_{\mathbf{A}}>0$ is the constant satisfying (3.23), and define the discrete version of $\mathscr{J}$ (cf. 3.15)):

$$
\mathscr{J}_{h}: \mathbf{K}_{h} \rightarrow \mathbf{K}_{h}, \quad \mathbf{w}_{h} \rightarrow \mathscr{J}_{h}\left(\mathbf{w}_{h}\right)=\mathbf{u}_{h}
$$

with $\mathbf{u}_{h} \in \mathbf{H}_{h, D}^{1}$ being the second component of $\left(\left(\mathbf{T}_{h, 0}, \mathbf{u}_{h}\right), \mu_{h}\right) \in \mathbf{X}_{h, 0} \times \mathbb{R}$, solution to

$$
\begin{array}{ll}
\mathbf{A}\left(\left(\mathbf{T}_{h, 0}, \mathbf{u}_{h}\right),\left(\mathbf{S}_{h}, \mathbf{v}_{h}\right)\right)+\mathbf{C}_{\mathbf{w}_{h}}\left(\left(\mathbf{T}_{h, 0}, \mathbf{u}_{h}\right),\left(\mathbf{S}_{h}, \mathbf{v}_{h}\right)\right)+\mathbf{B}\left(\left(\mathbf{S}_{h}, \mathbf{v}_{h}\right), \mu_{h}\right) & =\mathbf{F}\left(\mathbf{S}_{h}, \mathbf{v}_{h}\right), \\
\mathbf{B}\left(\left(\mathbf{T}_{h, 0}, \mathbf{u}_{h}\right), \eta_{h}\right) & =0,
\end{array}
$$

for all $\left(\left(\mathbf{S}_{h}, \mathbf{v}_{h}\right), \eta_{h}\right) \in \mathbf{X}_{h, 0} \times \mathbb{R}$. Similarly to the continuous case, the following equivalences hold:

$$
\mathscr{J}\left(\mathbf{u}_{h}\right)=\mathbf{u}_{h} \Leftrightarrow\left(\left(\mathbf{T}_{h, 0}, \mathbf{u}_{h}\right), \mu_{h}\right) \in \mathbf{X}_{h, 0} \times \mathbb{R} \text { satisfies 4.5) } \Leftrightarrow\left(\mathbf{T}_{h, 0}+\mu_{h} \mathrm{I}, \mathbf{u}_{h}\right) \in \mathbf{X}_{h} \text { satisfies 4.3. }
$$

Lemma 4.1 Assume that (H.O) and (H.1) hold and that $\kappa_{1}>0$ and $0<\kappa_{2}<4 v$. Assume further that the external forces $\mathbf{f}$ and $\mathbf{g}$ satisfy

$$
\left(4 v^{2}+\kappa_{1}^{2}\right)^{1 / 2}\|\mathbf{f}\|_{0, \Omega}+2 v\|\mathbf{g}\|_{-1 / 2,00, \Gamma_{N}} \leqslant \frac{\alpha_{\mathbf{A}}^{2}}{4 C_{\mathbf{C}}}
$$

with $\alpha_{\mathbf{A}}$ and $C_{\mathbf{C}}$ being the constants in 3.23 and 3.21, respectively. Then, given $\mathbf{w}_{h} \in \mathbf{K}_{h}$, there exists a unique $\mathbf{u}_{h} \in \mathbf{K}_{h}$, such that $\mathscr{J}\left(\mathbf{w}_{h}\right)=\mathbf{u}_{h}$. 
Proof. Similarly to the proof of Lemma 3.3 it is easy to see that, under hypothesis 4.11 , for all $\mathbf{w}_{h} \in \mathbf{K}_{h}$ the bilinear form $\mathbf{A}(\cdot, \cdot)+\mathbf{C}_{\mathbf{w}_{h}}(\cdot, \cdot)$ is elliptic on $\mathbf{X}_{h, 0}$, that is

$$
\mathbf{A}\left(\left(\mathbf{S}_{h}, \mathbf{v}_{h}\right),\left(\mathbf{S}_{h}, \mathbf{v}_{h}\right)\right)+\mathbf{C}_{\mathbf{w}_{h}}\left(\left(\mathbf{S}_{h}, \mathbf{v}_{h}\right),\left(\mathbf{S}_{h}, \mathbf{v}_{h}\right)\right) \geqslant \frac{\alpha_{\mathbf{A}}}{2}\left\|\left(\mathbf{S}_{h}, \mathbf{v}_{h}\right)\right\|_{\mathbf{X}}^{2},
$$

for all $\left(\mathbf{S}_{h}, \mathbf{v}_{h}\right) \in \mathbf{X}_{h, 0}$. Then, recalling that $\mathbf{B}$ satisfies 4.6 , the result is a direct consequence of the classical Babuška-Brezzi theory.

We are now in position of establishing the well-posedness of problem 4.3.

THEOREM 4.1 Assume that (H.0) and (H.1) hold and that $\kappa_{1}>0$ and $0<\kappa_{2}<4 v$. Assume further that the external forces $\mathbf{f}$ and $\mathbf{g}$ satisfy

$$
\left(4 v^{2}+\kappa_{1}^{2}\right)^{1 / 2}\|\mathbf{f}\|_{0, \Omega}+2 v\|\mathbf{g}\|_{-1 / 2,00, \Gamma_{N}}<\frac{\alpha_{\mathbf{A}}^{2}}{4 C_{\mathbf{C}}}
$$

with $\alpha_{\mathbf{A}}$ and $C_{\mathbf{C}}$ being the constants in $(3.23)$ and $(3.21)$, respectively. Then, there exists a unique $\left(\mathbf{T}_{h}, \mathbf{u}_{h}\right) \in \mathbf{X}_{h}$ solution to 4.3 . In addition, the solution $\left(\mathbf{T}_{h}, \mathbf{u}_{h}\right)$ satisfies the estimate

$$
\left\|\left(\mathbf{T}_{h}, \mathbf{u}_{h}\right)\right\|_{\mathbf{X}} \leqslant 2\left(\frac{1}{\alpha_{\mathbf{A}}}+n^{1 / 2}|\Omega|^{1 / 2}\left(\frac{1}{\tilde{\beta}}+\frac{C_{\mathbf{A}}}{\tilde{\beta} \alpha_{\mathbf{A}}}\right)\right)\left(\left(4 v^{2}+\kappa_{1}^{2}\right)^{1 / 2}\|\mathbf{f}\|_{0, \Omega}+2 v\|\mathbf{g}\|_{-1 / 2,00, \Gamma_{N}}\right) .
$$

Proof. According to 4.10, it is clear that for proving the existence and uniqueness of solution of problem (4.3) it suffices to prove that $\mathscr{J}_{h}$ posses a unique fixed-point in $\mathbf{K}_{h}$. To do that, we proceed analogously to the proof of Theorem 3.1 that is, we make use of hypothesis (4.13) to deduce that $\mathscr{J}_{h}$ is a contraction mapping in $\mathbf{K}_{h}$ and apply the well known Banach's fixed point Theorem to complete the result. Since that the deduction is straightforward, we omit further details. In turn, by applying the ellipticity of $\mathbf{A}(\cdot, \cdot)+\mathbf{C}_{\mathbf{w}_{h}}(\cdot, \cdot)$, with $\mathbf{w}_{h}=\mathbf{u}_{h}$, the inf-sup condition [4.6, and proceeding exactly as in the proof Theorem 3.1 we can obtain

$$
\left\|\mathbf{u}_{h}\right\|_{1, \Omega} \leqslant\left\|\left(\mathbf{T}_{h, 0}, \mathbf{u}_{h}\right)\right\| \mathbf{x} \leqslant \frac{2}{\alpha_{\mathbf{A}}}\left\|\mathbf{F} \mid \mathbf{X}_{h, 0}\right\|_{\mathbf{X}_{h, 0}^{\prime}} \quad \text { and } \quad\left|\mu_{h}\right| \leqslant \frac{2}{\beta}\left(1+\frac{C_{\mathbf{A}}}{\alpha_{\mathbf{A}}}\right)\left\|\left.\mathbf{F}\right|_{\mathbf{X}_{h, 0}}\right\| \mathbf{X}_{h, 0}^{\prime},
$$

which together with the fact that

$$
\left\|\left.\mathbf{F}\right|_{\mathbf{X}_{h, 0}}\right\|_{\mathbf{X}_{h, 0}^{\prime}} \leqslant\left(4 v^{2}+\kappa_{1}^{2}\right)^{1 / 2}\|\mathbf{f}\|_{0, \Omega}+2 v\|\mathbf{g}\|_{-1 / 2,00, \Gamma_{N}}
$$

yield (4.14).

\subsection{Céa’s estimate}

THEOREM 4.2 Assume that hypotheses of Theorem 4.1 hold. Let $(\mathbf{T}, \mathbf{u}) \in \mathbf{X}$ and $\left(\mathbf{T}_{h}, \mathbf{u}_{h}\right) \in \mathbf{X}_{h}$ be the unique solutions of the continuous and discrete problems 3.10) and 4.3. Then, there exists $C_{c e a}>0$, independent of $h$, such that

$$
\left\|(\mathbf{T}, \mathbf{u})-\left(\mathbf{T}_{h}, \mathbf{u}_{h}\right)\right\|_{\mathbf{X}} \leqslant C_{c e a} \inf _{\left(\mathbf{S}_{h}, \mathbf{v}_{h}\right) \in \mathbf{X}_{h}}\left\|(\mathbf{T}, \mathbf{u})-\left(\mathbf{S}_{h}, \mathbf{v}_{h}\right)\right\|_{\mathbf{X}}
$$

Proof. $\quad$ Let, $\mu \in \mathbb{R}, \mathbf{T}_{0} \in \mathbb{H}_{0}(\operatorname{div} ; \Omega)$ and $\mu_{h} \in \mathbb{R}, \mathbf{T}_{h, 0} \in \mathbb{H}_{h, 0}$, such that $\mathbf{T}=\mathbf{T}_{0}+\mu \mathrm{I}$ and $\mathbf{T}_{h}=\mathbf{T}_{h, 0}+\mu_{h} \mathrm{I}$, respectively, and define

$$
\boldsymbol{\phi}=\left(\mathbf{T}_{0}, \mathbf{u}\right) \in \mathbf{X}_{0}, \quad \boldsymbol{\phi}_{h}=\left(\mathbf{T}_{h, 0}, \mathbf{u}_{h}\right) \in \mathbf{X}_{h, 0}, \quad \mathbf{e}_{\boldsymbol{\phi}}=\boldsymbol{\phi}-\boldsymbol{\phi}_{h} \quad \text { and } \quad \mathrm{e}_{\mu}=\mu-\mu_{h} .
$$

According to 4.4, in what follows we equivalently prove that there exists $C>0$, independent of $h$, such that

$$
\left\|\mathbf{e}_{\boldsymbol{\phi}}\right\|_{\mathbf{X}}+\left|\mathrm{e}_{\mu}\right| \leqslant C\left\{\left\|\boldsymbol{\phi}-\psi_{h}\right\|_{\mathbf{X}}+\left|\mu-\eta_{h}\right|\right\} \quad \forall \psi_{h} \in \mathbf{X}_{h, 0}, \forall \eta_{h} \in \mathbb{R} .
$$

To do that we let,

$$
\operatorname{Ker}_{h}(\mathbf{B}):=\left\{\psi_{h}:=\left(\mathbf{S}_{h}, \mathbf{v}_{h}\right) \in \mathbf{X}_{h, 0}: \mathbf{B}\left(\psi_{h}, \eta_{h}\right)=0 \quad \forall \eta_{h} \in \mathbb{R}\right\},
$$

and recall that the following identity holds

$$
\inf _{\boldsymbol{\psi}_{h} \in \operatorname{Ker}_{h}(\mathbf{B})}\left\|\boldsymbol{\phi}-\psi_{h}\right\| \mathbf{x} \leqslant C\left\|\boldsymbol{\phi}-\psi_{h}\right\| \mathbf{x} \quad \forall \psi_{h} \in \mathbf{X}_{h, 0},
$$

with $C>0$ independent of $h$ (Gatica, 2014, estimate (2.89)). 
First, given arbitrary $\hat{\psi}_{h}:=\left(\hat{\mathbf{S}}_{h}, \hat{\mathbf{v}}_{h}\right) \in \operatorname{Ker}_{h}(\mathbf{B})$ and $\hat{\eta}_{h} \in \mathbb{R}$, let us decompose $\mathbf{e}_{\boldsymbol{\phi}}$ and $\mathrm{e}_{\mu}$ into

$$
\begin{array}{llll}
\mathbf{e}_{\boldsymbol{\phi}}=\boldsymbol{\xi}_{\boldsymbol{\phi}}+\chi_{\boldsymbol{\phi}} & \text { with } \quad \boldsymbol{\xi}_{\boldsymbol{\phi}}:=\boldsymbol{\phi}-\hat{\psi}_{h}, & \chi_{\boldsymbol{\phi}}:=\hat{\psi}_{h}-\boldsymbol{\phi}_{h}, \\
\mathrm{e}_{\mu}=\xi_{\mu}+\chi_{\mu} & \text { with } \quad \xi_{\mu}:=\mu-\hat{\eta}_{h}, \quad \chi_{\mu}:=\hat{\eta}_{h}-\mu_{h} .
\end{array}
$$

In turn, recalling that $(\boldsymbol{\phi}, \mu) \in \mathbf{X}_{0} \times \mathbb{R}$ and $\left(\boldsymbol{\phi}_{h}, \mu_{h}\right) \in \mathbf{X}_{h, 0} \times \mathbb{R}$ are solutions of 3.12) and 4.5, respectively (cf. (3.17), 4.10), by subtracting the first equations of 3.12) and 4.5, we readily get

$$
\mathbf{A}\left(\mathbf{e}_{\boldsymbol{\phi}}, \psi_{h}\right)+\left[\mathbf{C}_{\mathbf{u}}\left(\boldsymbol{\phi}, \psi_{h}\right)-\mathbf{C}_{\mathbf{u}_{h}}\left(\boldsymbol{\phi}_{h}, \psi_{h}\right)\right]+\mathbf{B}\left(\psi_{h}, \mathrm{e}_{\mu}\right)=0 \quad \forall \psi_{h}=\left(\mathbf{S}_{h}, \mathbf{v}_{h}\right) \in \mathbf{X}_{h, 0},
$$

which, after straightforward manipulations, yields

$$
\mathbf{A}\left(\mathbf{e}_{\boldsymbol{\phi}}, \psi_{h}\right)+\mathbf{C}_{\mathbf{u}_{h}}\left(\mathbf{e}_{\boldsymbol{\phi}}, \psi_{h}\right)+\mathbf{C}_{\mathbf{u}-\mathbf{u}_{h}}\left(\boldsymbol{\phi}, \psi_{h}\right)+\mathbf{B}\left(\psi_{h}, \mathrm{e}_{\mu}\right)=0 \quad \forall \psi_{h}=\left(\mathbf{S}_{h}, \mathbf{v}_{h}\right) \in \mathbf{X}_{h, 0} .
$$

In particular, taking $\psi_{h}=\boldsymbol{\chi}_{\boldsymbol{\phi}} \in \operatorname{Ker}_{h}(\mathbf{B})$ in 4.22, and using 4.20, it follows that

$$
\mathbf{A}\left(\boldsymbol{\chi}_{\boldsymbol{\phi}}, \boldsymbol{\chi}_{\boldsymbol{\phi}}\right)+\mathbf{C}_{\mathbf{u}_{h}}\left(\boldsymbol{\chi}_{\boldsymbol{\phi}}, \boldsymbol{\chi}_{\boldsymbol{\phi}}\right)=-\mathbf{A}\left(\boldsymbol{\xi}_{\boldsymbol{\phi}}, \boldsymbol{\chi}_{\boldsymbol{\phi}}\right)-\mathbf{C}_{\mathbf{u}-\mathbf{u}_{h}}\left(\boldsymbol{\phi}, \boldsymbol{\chi}_{\boldsymbol{\phi}}\right)-\mathbf{C}_{\mathbf{u}_{h}}\left(\boldsymbol{\xi}_{\boldsymbol{\phi}}, \boldsymbol{\chi}_{\boldsymbol{\phi}}\right)
$$

and then, using the ellipticity of $\mathbf{A}(\cdot, \cdot)+\mathbf{C}_{\mathbf{u}_{h}}(\cdot, \cdot)$ on $\mathbf{X}_{h, 0}$ (cf. 4.12), and employing the continuity of $\mathbf{A}$ and $\mathbf{C}_{\mathbf{w}}$, with $\mathbf{w}=\mathbf{u}-\mathbf{u}_{h}$, and $\mathbf{w}=\mathbf{u}_{h}$, (cf. 3.20) and 3.21), and the fact that $\left\|\mathbf{u}-\mathbf{u}_{h}\right\|_{1, \Omega} \leqslant\left\|\boldsymbol{\xi}_{\boldsymbol{\phi}}\right\| \mathbf{x}+\left\|\boldsymbol{\chi}_{\boldsymbol{\phi}}\right\| \mathbf{x}$, we obtain

$$
\frac{\alpha_{\mathbf{A}}}{2}\left\|\boldsymbol{\chi}_{\boldsymbol{\phi}}\right\|_{\mathbf{X}} \leqslant\left(C_{\mathbf{A}}+C_{\mathbf{C}}\left\|\mathbf{u}_{h}\right\|_{1, \Omega}+C_{\mathbf{C}}\|\boldsymbol{\phi}\|_{\mathbf{X}}\right)\left\|\boldsymbol{\xi}_{\boldsymbol{\phi}}\right\|_{\mathbf{x}}+C_{\mathbf{C}}\|\boldsymbol{\phi}\|_{\mathbf{X}}\left\|\boldsymbol{\chi}_{\boldsymbol{\phi}}\right\|_{\mathbf{X}}
$$

which together with 3.34 and 4.15 , imply

$$
\left(\frac{\alpha_{\mathbf{A}}}{2}-C_{\mathbf{C}}\left\|\left.\mathbf{F}\right|_{\mathbf{X}_{0}}\right\|_{\mathbf{X}_{0}^{\prime}}\right)\left\|\boldsymbol{\chi}_{\boldsymbol{\phi}}\right\|_{\mathbf{X}} \leqslant\left(C_{\mathbf{A}}+\frac{2 C_{\mathbf{C}}}{\alpha_{\mathbf{A}}}\left\|\left.\mathbf{F}\right|_{\mathbf{X}_{h, 0}}\right\|_{\mathbf{X}_{h, 0}^{\prime}}+\frac{2 C_{\mathbf{C}}}{\alpha_{\mathbf{A}}}\left\|\left.\mathbf{F}\right|_{\mathbf{X}_{0}}\right\|_{\mathbf{X}_{0}^{\prime}}\right)\left\|\boldsymbol{\xi}_{\boldsymbol{\phi}}\right\| \mathbf{x}
$$

The latter, along with assumption 4.13, estimates 3.22, 4.16 and the triangle inequality, imply that there exists $C_{1}>0$, independent of $h$, such that

$$
\left\|\mathbf{e}_{\boldsymbol{\phi}}\right\|_{\mathbf{x}} \leqslant\left\|\boldsymbol{\xi}_{\boldsymbol{\phi}}\right\|_{\mathbf{x}}+\left\|\boldsymbol{\chi}_{\boldsymbol{\phi}}\right\|_{\mathbf{x}} \leqslant C_{1}\left\|\boldsymbol{\xi}_{\boldsymbol{\phi}}\right\|_{\mathbf{x}}
$$

On the other hand, using the inf-sup condition 4.6) and 4.22), it is easy to see that

$$
\tilde{\beta}\left|\chi_{\mu}\right| \leqslant \sup _{\boldsymbol{\psi}_{h} \in \mathbf{X}_{h, 0} \backslash\{\mathbf{0}\}} \frac{\mathbf{B}\left(\psi_{h}, \chi_{\mu}\right)}{\left\|\psi_{h}\right\| \mathbf{X}}=\sup _{\boldsymbol{\psi}_{h} \in \mathbf{X}_{h, 0} \backslash\{\mathbf{0}\}} \frac{-\mathbf{A}\left(\mathbf{e}_{\boldsymbol{\phi}}, \psi_{h}\right)-\mathbf{C}_{\mathbf{u}_{h}}\left(\mathbf{e}_{\boldsymbol{\phi}}, \psi_{h}\right)-\mathbf{C}_{\mathbf{u}-\mathbf{u}_{h}}\left(\boldsymbol{\phi}, \psi_{h}\right)-\mathbf{B}\left(\psi_{h}, \xi_{\mu}\right)}{\left\|\psi_{h}\right\|_{\mathbf{X}}},
$$

which combined with the continuity of $\mathbf{A}, \mathbf{B}$ and $\mathbf{C}_{\mathbf{w}}$, with $\mathbf{w}=\mathbf{u}_{h}$ and $\mathbf{w}=\mathbf{u}-\mathbf{u}_{h}$, and estimates 3.34, 4.15, and 4.24 , yield

which clearly implies

$$
\left|\chi_{\mu}\right| \leqslant C\left\{\left\|\boldsymbol{\xi}_{\phi}\right\|_{\mathbf{x}}+\left|\xi_{\mu}\right|\right\}
$$

$$
\left|\mathrm{e}_{\mu}\right| \leqslant C_{2}\left\{\left\|\boldsymbol{\xi}_{\boldsymbol{\phi}}\right\| \mathbf{x}+\left|\boldsymbol{\xi}_{\mu}\right|\right\} .
$$

Therefore, from 4.19, 4.24, 4.25 and the fact that $\hat{\psi}_{h} \in \operatorname{Ker}_{h}(\mathbf{B})$ and $\hat{\eta}_{h} \in \mathbb{R}$ are arbitrary we easily obtain 4.18) and conclude the proof.

We end this section by observing that, provided the solution $\left(\mathbf{T}_{h}, \mathbf{u}_{h}\right) \in \mathbf{X}_{h}$ of problem 4.3, we can approximate the pressure $p$ and the shear stress $\boldsymbol{\sigma}$ by using the following post-processing formulas:

$$
p_{h}=-\frac{1}{n}\left(\operatorname{tr}\left(\mathbf{T}_{h}\right)+\operatorname{tr}\left(\mathbf{u}_{h} \otimes \mathbf{u}_{h}\right)\right) \quad \text { and } \quad \boldsymbol{\sigma}_{h}=\mathbf{T}_{h}+\mathbf{u}_{h} \otimes \mathbf{u}_{h} .
$$

The following corollary establishes the approximation result for this post-processing procedure.

Corollary 4.1 Assume that the hypotheses of Theorem 4.1 hold true. Let $(\mathbf{T}, \mathbf{u}) \in \mathbf{X}$ and $\left(\mathbf{T}_{h}, \mathbf{u}_{h}\right) \in \mathbf{X}_{h}$ be the unique solutions of the continuous and discrete problems (3.10) and 4.3, respectively. Let $p_{h}$ and $\boldsymbol{\sigma}_{h}$ be given by 4.26 . Then there exists $\widehat{C}>0$, independent of $h$, such that

$$
\left\|p-p_{h}\right\|_{0, \Omega}+\left\|\boldsymbol{\sigma}-\boldsymbol{\sigma}_{h}\right\|_{0, \Omega} \leqslant \widehat{C} \inf _{\left(\mathbf{S}_{h}, \mathbf{v}_{h}\right) \in \mathbf{X}_{h}}\left\|(\mathbf{T}, \mathbf{u})-\left(\mathbf{S}_{h}, \mathbf{v}_{h}\right)\right\| \mathbf{X}
$$

Proof. Using estimates 3.35, 4.15) and the fact that $H^{1}$ is continuously embedded into $L^{4}$, it is not difficult to see that

$$
\begin{aligned}
\left\|\mathbf{u} \otimes \mathbf{u}-\mathbf{u}_{h} \otimes \mathbf{u}_{h}\right\|_{0, \Omega} & \leqslant\left\|\left(\mathbf{u}-\mathbf{u}_{h}\right) \otimes \mathbf{u}\right\|_{0, \Omega}+\left\|\mathbf{u}_{h} \otimes\left(\mathbf{u}-\mathbf{u}_{h}\right)\right\|_{0, \Omega} \\
& \leqslant C\left\{\left\|\left(\mathbf{u}-\mathbf{u}_{h}\right)\right\|_{1, \Omega}\|\mathbf{u}\|_{1, \Omega}+\left\|\left(\mathbf{u}-\mathbf{u}_{h}\right)\right\|_{1, \Omega}\left\|\mathbf{u}_{h}\right\|_{1, \Omega}\right\} \\
& \leqslant \hat{C}\left\|\left(\mathbf{u}-\mathbf{u}_{h}\right)\right\|_{1, \Omega} .
\end{aligned}
$$

Then, the result follows from 4.17, 4.28, and equations 2.3, 2.4) and 4.26. 
TABLE 1. Local degrees of freedom for the lowestorder method $(k=0)$.

\begin{tabular}{l|c|c|c} 
& Bernardi-Raugel & MINI-element & $\mathbf{R T}_{0}-P_{1}$ \\
\hline local Dof & 10 & 11 & 12
\end{tabular}

\subsection{A particular choice of discrete spaces}

Let $\mathscr{T}_{h}$ be a regular family of triangulations of the polyhedral region $\bar{\Omega}$ by triangles $K$ in $\mathbb{R}^{2}$ or tetrahedra in $\mathbb{R}^{3}$ of diameter $h_{K}$ such that $\bar{\Omega}=\cup\left\{K: K \in \mathscr{T}_{h}\right\}$ and define $h:=\max \left\{h_{K}: K \in \mathscr{T}_{h}\right\}$. For each integer $k \geqslant 0$ and for each $K \in \mathscr{T}_{h}$, we define the local Raviart-Thomas space of order $k$ (see, for instance Brezzi \& Fortin. 1991):

$$
\mathbf{R T}_{k}(K):=\mathbf{P}_{k}(K) \oplus P_{k}(K) \mathbf{x}
$$

where $\mathbf{x}:=\left(x_{1}, \ldots, x_{n}\right)^{t}$ is a generic vector of $\mathbb{R}^{n}$. Then, we specify the discrete spaces in 4.1 by:

$$
\begin{aligned}
& \mathbf{H}_{h}(\operatorname{div} ; \Omega):=\left\{\mathbf{s}=\left(s_{1}, \ldots, s_{n}\right) \in \mathbf{H}(\operatorname{div} ; \Omega):\left.\quad \mathbf{s}\right|_{K} \in \mathbf{R T}_{k}(K), \quad \forall K \in \mathscr{T}_{h}\right\}, \\
& \mathrm{H}_{h, D}^{1}(\Omega):=\left\{v \in C(\bar{\Omega}):\left.\quad v\right|_{K} \in P_{k+1}(K), \quad \forall K \in \mathscr{T}_{h} \quad \text { and }\left.\quad v\right|_{\Gamma_{D}}=0\right\} .
\end{aligned}
$$

It is not difficult to see that these spaces satisfy hypotheses (H.0) and (H.1) (see Section 4.1). In particular, (H.1) is easy to verify if the sequence of subspaces is nested or if we are able to find a coarser space where (H.1) holds. Then, $\hat{\mathbf{v}}_{0}$ can be constructed exactly as explained at the end of the proof of Lemma 3.2 In turn, it is well known that these subspaces satisfy the following approximation properties (see, e.g. Ciarlet 1978 , Gatica, 2014):

For each $r>0$ and for each $\mathbf{s}=\left(s_{1}, \ldots, s_{n}\right) \in \mathbf{H}^{r}(\Omega)$, with $\operatorname{div} \mathbf{s} \in \mathrm{H}^{r}(\Omega)$, there exists $\mathbf{s}_{h} \in \mathbf{H}_{h}(\operatorname{div} ; \Omega)$, such that

$$
\left\|\mathbf{S}-\mathbf{s}_{h}\right\|_{\operatorname{div}, \Omega} \leqslant C h^{\min \{r, k+1\}}\left\{\|\mathbf{s}\|_{r, \Omega}+\|\operatorname{div} \mathbf{s}\|_{r, \Omega}\right\} .
$$

For each $r>0$ and for each $v \in \mathrm{H}^{r+1}(\Omega) \cap \mathrm{H}_{\Gamma_{D}}^{1}(\Omega)$ there exists $v_{h} \in \mathrm{H}_{h, D}^{1}(\Omega)$ such that

$$
\left\|v-v_{h}\right\|_{1, \Omega} \leqslant C h^{\min \{r, k+1\}}\|v\|_{r+1, \Omega} .
$$

The theoretical rate of convergence for 4.3 with spaces as in 4.29 is stated in the following result.

THeOREM 4.3 Let $\mathbf{X}_{h}=\mathbb{H}_{h} \times \mathbf{H}_{h, D}^{1}$ defined in terms of the spaces 4.29 . Let $(\mathbf{T}, \mathbf{u}) \in \mathbf{X}$ and $\left(\mathbf{T}_{h}, \mathbf{u}_{h}\right) \in \mathbf{X}_{h}$ be the unique solutions of the continuous and discrete problems $(3.10)$ and $(4.3)$, respectively. In addition to the hypotheses of Theorem 4.1 let us assume that $\mathbf{T} \in \mathbb{H}^{r}(\Omega), \operatorname{div} \mathbf{T} \in \mathbf{H}^{r}(\Omega)$, and $\mathbf{u} \in \mathbf{H}^{r+1}(\Omega)$, for some $r>0$. Then, there exists $C_{\text {rate }}>0$, independent of $h$, such that

$$
\left\|(\mathbf{T}, \mathbf{u})-\left(\mathbf{T}_{h}, \mathbf{u}_{h}\right)\right\| \mathbf{x} \leqslant C_{\text {rate }} h^{\min \{r, k+1\}}\left\{\|\mathbf{T}\|_{r, \Omega}+\|\operatorname{div} \mathbf{T}\|_{r, \Omega}+\|\mathbf{u}\|_{r+1, \Omega}\right\}
$$

Proof. The result is a straightforward application of Theorem 4.2 and properties 4.30) and 4.31.

We now provide the rate of convergence for the post-processing introduced in 4.26 .

COROLlary 4.2 There exists $\widetilde{C}>0$, independent of $h$, such that

$$
\left\|p-p_{h}\right\|_{0, \Omega}+\left\|\boldsymbol{\sigma}-\boldsymbol{\sigma}_{h}\right\|_{0, \Omega}+\leqslant \widetilde{C} h^{\min \{r, k+1\}}\left\{\|\mathbf{T}\|_{r, \Omega}+\|\operatorname{div} \mathbf{T}\|_{r, \Omega}+\|\mathbf{u}\|_{r+1, \Omega}\right\} .
$$

Proof. The result is a direct application of Theorem 4.3 and Corollary 4.1

REMARK 4.1 In Table 1 we compare the local degrees of freedom (Dof) of our method, considering $k=0$ and $n=2$, with the corresponding local Dof of the velocity-pressure formulation discretized by the Bernardi-Raugel element and the MINI-element (see Chapter III in Girault \& Raviart (1986)). We observe there that, although our formulation possesses considerably more unknowns ( 6 unknowns in 2D) than the velocity-pressure formulation (3 unknowns in 2D), the computational cost is not considerably increased. 


\section{A posteriori error analysis}

For each $K \in \mathscr{T}_{h}$ we let $\mathscr{E}(K)$ be the set of edges (faces) of $K$ and we denote by $\mathscr{E}_{h}$ the set of all edges (faces) of $\mathscr{T}_{h}$, subdivided as follows:

$$
\mathscr{E}_{h}=\mathscr{E}_{h}(\Omega) \cup \mathscr{E}_{h}\left(\Gamma_{D}\right) \cup \mathscr{E}_{h}\left(\Gamma_{N}\right),
$$

where $\mathscr{E}_{h}(\Omega):=\left\{e \in \mathscr{E}_{h}: e \subseteq \Omega\right\}, \mathscr{E}_{h}\left(\Gamma_{D}\right):=\left\{e \in \mathscr{E}_{h}: e \subseteq \Gamma_{D}\right\}$ and $\mathscr{E}_{h}\left(\Gamma_{N}\right):=\left\{e \in \mathscr{E}_{h}: e \subseteq \Gamma_{N}\right\}$. In what follows, $h_{e}$ stands for the diameter of a given edge (face) $e \in \mathscr{E}_{h}$. Also, we fix a unit normal vector $\mathbf{n}_{e}$ to the edge (face) $e$ (its particular orientation is not relevant). However, when no confusion arises, we will simply write $\mathbf{n}$ instead of $\mathbf{n}_{e}$.

Now, let $(\mathbf{T}, \mathbf{u}) \in \mathbf{X}$ and $\left(\mathbf{T}_{h}, \mathbf{u}_{h}\right) \in \mathbf{X}_{h}$ be the unique solutions of the continuous and discrete problems 3.10) and 4.3, respectively. Then, we introduce the global a posteriori error estimator

$$
\Theta:=\left\{\sum_{K \in \mathscr{T} h} \Theta_{K}^{2}\right\}^{1 / 2},
$$

defined locally (for each $K \in \mathscr{T}_{h}$ ) as

$$
\Theta_{K}^{2}:=\left\|\mathbf{f}+\operatorname{div} \mathbf{T}_{h}\right\|_{0, K}^{2}+\left\|\mathbf{T}_{h}^{\mathrm{d}}-2 v \mathbf{e}\left(\mathbf{u}_{h}\right)+\left(\mathbf{u}_{h} \otimes \mathbf{u}_{h}\right)^{\mathrm{d}}\right\|_{0, K}^{2}+\sum_{e \in \mathscr{E}(K) \cap \mathscr{E}_{h}\left(\Gamma_{N}\right)} h_{e}\left\|\mathbf{g}-\mathbf{T}_{h} \mathbf{n}-\left(\mathbf{u}_{h} \otimes \mathbf{u}_{h}\right) \mathbf{n}\right\|_{0, e}^{2} .
$$

\subsection{Reliability of the a posteriori error estimator}

The main result of this section is stated as follows

Theorem 5.1 Let $(\mathbf{T}, \mathbf{u}) \in \mathbf{X}$ and $\left(\mathbf{T}_{h}, \mathbf{u}_{h}\right) \in \mathbf{X}_{h}$ be the unique solutions of the continuous and discrete problems 3.10 and 4.3 , respectively. Assume that $\mathbf{g} \in \mathbf{L}^{2}\left(\Gamma_{N}\right)$ and that

$$
\left(4 v^{2}+\kappa_{1}^{2}\right)^{1 / 2}\|\mathbf{f}\|_{0, \Omega}+2 v\|\mathbf{g}\|_{-1 / 2,00, \Gamma_{N}} \leqslant \frac{C_{\mathrm{glob}}}{4 C_{\mathbf{C}}}\left(\frac{1}{\alpha_{\mathbf{A}}}+n^{1 / 2}|\Omega|^{1 / 2}\left(\frac{1}{\tilde{\beta}}+\frac{C_{\mathbf{A}}}{\tilde{\beta} \alpha_{\mathbf{A}}}\right)\right)^{-1}
$$

with $C_{\mathrm{glob}}$ being the positive constant in 5.6 below. Then, there exists $C_{\mathrm{rel}}>0$, independent of $h$, such that

$$
\left\|\left(\mathbf{T}-\mathbf{T}_{h}, \mathbf{u}-\mathbf{u}_{h}\right)\right\|_{\mathbf{x}} \leqslant C_{\mathrm{rel}} \Theta .
$$

We begin the derivation of (5.4) by observing that, since $\left(\mathbf{A}+\mathbf{C}_{\mathbf{u}}\right)(\cdot, \cdot)$ is elliptic on $\mathbf{X}_{0}$ and $\mathbf{B}$ satisfies the inf-sup condition (3.24), then the following global inf-sup condition holds (Ern \& Guermond 2004, Proposition 2.36)

$$
C_{\mathrm{glob}}\left(\|(\mathbf{R}, \mathbf{z})\|_{\mathbf{X}}+|\lambda|\right) \leqslant \sup _{((\mathbf{S}, \mathbf{v}), \eta) \in\left(\mathbf{X}_{0} \times \mathbb{R}\right) \backslash\{\mathbf{0}\}} \frac{\left(\mathbf{A}+\mathbf{C}_{\mathbf{u}}\right)((\mathbf{R}, \mathbf{z}),(\mathbf{S}, \mathbf{v}))+\mathbf{B}((\mathbf{R}, \mathbf{z}), \eta)+\mathbf{B}((\mathbf{S}, \mathbf{v}), \lambda)}{\|(\mathbf{S}, \mathbf{v})\| \mathbf{x}+|\eta|},
$$

for all $((\mathbf{R}, \mathbf{z}), \lambda) \in \mathbf{X}_{0} \times \mathbb{R}$, with

$$
C_{\mathrm{glob}}:=\frac{\alpha_{\mathbf{A}} \beta^{2}}{\left(\alpha_{\mathbf{A}}+\beta+C_{\mathbf{C}}+C_{\mathbf{A}}\right)\left(1+\beta+C_{\mathbf{C}}+C_{\mathbf{A}}\right)},
$$

which according to 1.1 , implies

$$
C_{\text {glob }}\|(\mathbf{R}, \mathbf{z})\|_{\mathbf{X}} \leqslant \sup _{(\mathbf{S}, \mathbf{v}) \in \mathbf{X} \backslash\{\mathbf{0}\}} \frac{\left(\mathbf{A}+\mathbf{C}_{\mathbf{u}}\right)((\mathbf{R}, \mathbf{z}),(\mathbf{S}, \mathbf{v}))}{\|(\mathbf{S}, \mathbf{v})\|_{\mathbf{X}}} \quad \forall(\mathbf{R}, \mathbf{z}) \in \mathbf{X} .
$$

REMARK 5.1 Recalling that $C_{\mathbf{A}}=4 \max \left\{1, \kappa_{1}, 2 v, 2 v \kappa_{2}, \kappa_{2}\right\}, C_{\mathbf{C}}=\|\mathbf{i}\|^{2}\left(1+\kappa_{2}^{2}\right)^{1 / 2}+2 v\left\|\mathbf{i}_{\Gamma}\right\|^{2}\left\|\gamma_{0}\right\|, \beta=$ $\frac{2 v\left\langle\mathbf{v}_{0} \cdot \mathbf{n}, 1\right\rangle}{\left\|\mathbf{v}_{0}\right\|_{1, \Omega}}$ and $\alpha_{\mathbf{A}}=\frac{1}{2} \min \left\{\min \left\{1, \kappa_{1}\right\}, \kappa_{1}, \kappa_{2}\left(4 v-\kappa_{2}\right) C_{K}\right\}$, taking $\kappa_{1}=\kappa_{2}=2 v$, for sufficiently small $v$ we observe that $\alpha_{\mathbf{A}} \approx v^{2}, C_{\mathbf{C}} \approx 1+v, \beta \approx v, C_{\mathbf{A}}$ can be seen as a constant independent of $v$, and then

$$
C_{\mathrm{glob}} \approx \frac{v^{4}}{\left(v^{2}+v+1\right)(1+v)} .
$$

Therefore, since $C_{\mathrm{rel}} \approx C_{\mathrm{glob}}^{-1}$, the reliability of the estimator $\Theta$ might be affected if $v$ is close to 0 (see 5.11) below). 
On the other hand, thanks to 3.10 and additional algebraic manipulations, we easily find that

$$
\left(\mathbf{A}+\mathbf{C}_{\mathbf{u}}\right)\left(\left(\mathbf{T}-\mathbf{T}_{h}, \mathbf{u}-\mathbf{u}_{h}\right),(\mathbf{S}, \mathbf{v})\right)=\mathbf{F}(\mathbf{S}, \mathbf{v})-\left(\mathbf{A}+\mathbf{C}_{\mathbf{u}_{h}}\right)\left(\left(\mathbf{T}_{h}, \mathbf{u}_{h}\right),(\mathbf{S}, \mathbf{v})\right)+\mathbf{C}_{\mathbf{u}_{h}-\mathbf{u}}\left(\left(\mathbf{T}_{h}, \mathbf{u}_{h}\right),(\mathbf{S}, \mathbf{v})\right) .
$$

In addition, we recall from 3.21 that

$$
\left|\mathbf{C}_{\mathbf{u}_{h}-\mathbf{u}}\left(\left(\mathbf{T}_{h}, \mathbf{u}_{h}\right),(\mathbf{S}, \mathbf{v})\right)\right| \leqslant C_{\mathbf{C}}\left\|\mathbf{u}_{h}-\mathbf{u}\right\|_{1, \Omega}\left\|\left(\mathbf{T}_{h}, \mathbf{u}_{h}\right)\right\|_{\mathbf{X}}\|(\mathbf{S}, \mathbf{v})\|_{\mathbf{X}},
$$

which thanks to (4.14) and (5.3), and the fact that $\left\|\mathbf{u}_{h}-\mathbf{u}\right\|_{1, \Omega} \leqslant\left\|\left(\mathbf{T}-\mathbf{T}_{h}, \mathbf{u}-\mathbf{u}_{h}\right)\right\| \mathbf{X}$, yields

$$
\left|\mathbf{C}_{\mathbf{u}_{h}-\mathbf{u}}\left(\left(\mathbf{T}_{h}, \mathbf{u}_{h}\right),(\mathbf{S}, \mathbf{v})\right)\right| \leqslant \frac{C_{\mathrm{glob}}}{2}\left\|\left(\mathbf{T}-\mathbf{T}_{h}, \mathbf{u}-\mathbf{u}_{h}\right)\right\|_{\mathbf{X}}\|(\mathbf{S}, \mathbf{v})\|_{\mathbf{X}} .
$$

Thus, taking $(\mathbf{R}, \mathbf{z})=\left(\mathbf{T}-\mathbf{T}_{h}, \mathbf{u}-\mathbf{u}_{h}\right)$ in (5.6) and using (5.7) and the previous inequality, we arrive at

$$
\frac{C_{\mathrm{glob}}}{2}\left\|\left(\mathbf{T}-\mathbf{T}_{h}, \mathbf{u}-\mathbf{u}_{h}\right)\right\| \mathbf{X} \quad \leqslant \sup _{(\mathbf{S}, \mathbf{v}) \in \mathbf{X} \backslash\{\mathbf{0}\}} \frac{|\mathscr{R}(\mathbf{S}, \mathbf{v})|}{\|(\mathbf{S}, \mathbf{v})\|_{\mathbf{X}}}
$$

where $\mathscr{R}: \mathbf{X} \rightarrow \mathbb{R}$ is the residual functional defined by

$$
\mathscr{R}(\mathbf{S}, \mathbf{v}):=\mathbf{F}(\mathbf{S}, \mathbf{v})-\left(\mathbf{A}+\mathbf{C}_{\mathbf{u}_{h}}\right)\left(\left(\mathbf{T}_{h}, \mathbf{u}_{h}\right),(\mathbf{S}, \mathbf{v})\right) \quad \forall(\mathbf{S}, \mathbf{v}) \in \mathbf{X} .
$$

More precisely, according to 2.6, 3.1, 3.5, 4.3) and the definitions of $\mathbf{A}, \mathbf{B}, \mathbf{C}_{\mathbf{u}_{h}}$ and $\mathbf{F}$ (cf. 3.8, 3.9), we find that for any $(\mathbf{S}, \mathbf{v}) \in \mathbf{X}$, there holds

$$
\mathscr{R}(\mathbf{S}, \mathbf{v})=\mathscr{R}_{1}(\mathbf{S})+\mathscr{R}_{2}(\mathbf{v}),
$$

where

$$
\mathscr{R}_{1}(\mathbf{S}):=-\kappa_{1}\left(\mathbf{f}+\operatorname{div} \mathbf{T}_{h}, \operatorname{div} \mathbf{S}\right)_{\Omega}-\left(\mathbf{T}_{h}^{\mathrm{d}}+2 v \boldsymbol{\omega}\left(\mathbf{u}_{h}\right)+\left(\mathbf{u}_{h} \otimes \mathbf{u}_{h}\right)^{\mathrm{d}}, \mathbf{S}\right)_{\Omega}-2 v\left(\mathbf{u}_{h}, \operatorname{div} \mathbf{S}\right)_{\Omega}+2 v\left\langle\mathbf{S n}, \mathbf{u}_{h}\right\rangle_{\Gamma_{N}},
$$

and

$$
\begin{aligned}
\mathscr{R}_{2}(\mathbf{v}):=\quad 2 v\left(\mathbf{f}+\operatorname{div}_{h}, \mathbf{v}\right)_{\Omega}+\kappa_{2}\left(\mathbf{T}_{h}^{\mathrm{d}}-2 v \mathbf{e}\left(\mathbf{u}_{h}\right)+\left(\mathbf{u}_{h} \otimes \mathbf{u}_{h}\right)^{\mathrm{d}}, \mathbf{e}(\mathbf{v})\right)_{\Omega} \\
+2 v\left(\mathbf{T}_{h}, \boldsymbol{\omega}(\mathbf{v})\right)_{\Omega}+2 v\left\langle\mathbf{g}-\mathbf{T}_{h} \mathbf{n}-\left(\mathbf{u}_{h} \otimes \mathbf{u}_{h}\right) \mathbf{n}, \mathbf{v}\right\rangle_{\Gamma_{N}} .
\end{aligned}
$$

Hence, the supremum in 5.8 can be bounded in terms of $\mathscr{R}_{1}$ and $\mathscr{R}_{2}$, which yields

$$
\frac{C_{\mathrm{glob}}}{2}\left\|\left(\mathbf{T}-\mathbf{T}_{h}, \mathbf{u}-\mathbf{u}_{h}\right)\right\|_{\mathbf{X}} \leqslant\left\|\mathscr{R}_{1}\right\|_{\mathbb{H}(\mathbf{d i v} ; \Omega)^{\prime}}+\left\|\mathscr{R}_{2}\right\|_{\mathbf{H}_{\Gamma_{D}^{1}}^{1}(\Omega)^{\prime}} .
$$

Throughout the rest of this section we provide suitable upper bounds for each one of the terms on the right hand side of 5.11). We begin with the upper bound of $\mathscr{R}_{1}$.

LEMMA 5.1 There holds

$$
\begin{aligned}
\left\|\mathscr{R}_{1}\right\|_{\mathbb{H}(\mathbf{d i v} ; \Omega)^{\prime}} & \leqslant\left\{\kappa_{1}^{2}\left\|\mathbf{f}+\operatorname{div} \mathbf{T}_{h}\right\|_{0, \Omega}^{2}+\left\|\mathbf{T}_{h}^{\mathrm{d}}-2 v \mathbf{e}\left(\mathbf{u}_{h}\right)+\left(\mathbf{u}_{h} \otimes \mathbf{u}_{h}\right)^{\mathrm{d}}\right\|_{0, \Omega}^{2}\right\}^{1 / 2} \\
& =\left\{\sum_{K \in \mathscr{T}_{h}} \kappa_{1}^{2}\left\|\mathbf{f}+\operatorname{div} \mathbf{T}_{h}\right\|_{0, K}^{2}+\left\|\mathbf{T}_{h}^{\mathrm{d}}-2 v \mathbf{e}\left(\mathbf{u}_{h}\right)+\left(\mathbf{u}_{h} \otimes \mathbf{u}_{h}\right)^{\mathrm{d}}\right\|_{0, K}^{2}\right\}^{1 / 2} .
\end{aligned}
$$

Proof. Similarly as in (Gatica et al. 2016. Section 3.3), we integrate by parts $\left(\operatorname{div} \mathbf{S}, \mathbf{u}_{h}\right)_{\Omega}$ and utilize the fact that $\mathbf{e}\left(\mathbf{u}_{h}\right)=\nabla \mathbf{u}_{h}-\boldsymbol{\omega}\left(\mathbf{u}_{h}\right)$, to observe that $\mathscr{R}_{1}$ can be rewritten as follows:

$$
\mathscr{R}_{1}(\mathbf{S})=-\kappa_{1}\left(\mathbf{f}+\operatorname{div} \mathbf{T}_{h}, \operatorname{div} \mathbf{S}\right)_{\Omega}-\left(\mathbf{T}_{h}^{\mathrm{d}}-2 v \mathbf{e}\left(\mathbf{u}_{h}\right)+\left(\mathbf{u}_{h} \otimes \mathbf{u}_{h}\right)^{\mathrm{d}}, \mathbf{S}\right)_{\Omega} .
$$

Then (5.12) follows straightforwardly from (5.13) and the Cauchy-Schwarz inequality.

To derive the estimate for $\mathscr{R}_{2}$ we need to introduce the well-known Clément operator $I_{h}: \mathrm{H}^{1}(\Omega) \rightarrow Y_{h}:=$ $\left\{v \in C(\bar{\Omega}):\left.v\right|_{K} \in P_{1}(K), \forall K \in \mathscr{T}_{h}\right\}$, which approximate optimally non-smooth functions by continuous piecewise linear functions. Of this operator, we will only use the following approximation properties (see Clément. 1975 for details): There exist constants $c_{1}, c_{2}>0$, independent of $h$, such that for all $v \in H^{1}(\Omega)$,

$$
\begin{array}{cl}
\left\|v-I_{h} v\right\|_{0, K} \leqslant c_{1} h_{K}\|v\|_{1, \Delta(K)} & \forall K \in \mathscr{T}_{h}, \\
\left\|v-I_{h} v\right\|_{0, e} \leqslant c h_{e}^{1 / 2}\|v\|_{1, \Delta(e)} & \forall e \in \mathscr{E}_{h},
\end{array}
$$

where

$$
\Delta(K):=\cup\left\{K^{\prime} \in \mathscr{T}_{h}: \quad K^{\prime} \cap K \neq \emptyset\right\} \quad \text { and } \quad \Delta(e):=\cup\left\{K^{\prime} \in \mathscr{T}_{h}: \quad K^{\prime} \cap e \neq \emptyset\right\} .
$$

The following lemma provides the upper bound for $\mathscr{R}_{2}$. 
Lemma 5.2 There exists $C>0$, independent of $h$, such that

$$
\left\|\mathscr{R}_{2}\right\|_{\mathbf{H}_{\Gamma_{D}}^{1}(\Omega)^{\prime}} \leqslant C\left\{\sum_{K \in \mathscr{T}_{h}} \widehat{\Theta}_{K}^{2}\right\}^{1 / 2},
$$

where, for each $K \in \mathscr{T}_{h}$ :

$$
\widehat{\Theta}_{K}^{2}:=h_{K}^{2}\left\|\mathbf{f}+\operatorname{div} \mathbf{T}_{h}\right\|_{0, K}^{2}+\left\|\mathbf{T}_{h}^{\mathrm{d}}-2 v \mathbf{e}\left(\mathbf{u}_{h}\right)+\left(\mathbf{u}_{h} \otimes \mathbf{u}_{h}\right)^{\mathrm{d}}\right\|_{0, K}^{2}+\sum_{e \in \mathscr{E}(K) \cap \mathscr{E}_{h}\left(\Gamma_{N}\right)} h_{e}\left\|\mathbf{g}-\mathbf{T}_{h} \mathbf{n}-\left(\mathbf{u}_{h} \otimes \mathbf{u}_{h}\right) \mathbf{n}\right\|_{0, e}^{2}
$$

Proof. Given $\mathbf{v} \in \mathbf{H}_{\Gamma_{D}}^{1}(\Omega)$, we set $\hat{\mathbf{v}}_{h}=\mathbf{I}_{h}(\mathbf{v}) \in \mathbf{H}_{h, D}^{1}$, with $\mathbf{I}_{h}$ being the vector version of $I_{h}$, which is defined componentwise by $I_{h}$, and observe from 4.3 with $\mathbf{S}_{h}=\mathbf{0}$ and the fact that $\frac{1}{2}\left(\operatorname{as}\left(\mathbf{T}_{h}\right), \operatorname{curl}\left(\hat{\mathbf{v}}_{h}\right)\right)_{\Omega}=$ $\left(\mathbf{T}_{h}, \boldsymbol{\omega}\left(\hat{\mathbf{v}}_{h}\right)\right)_{\Omega}$, that

$$
\begin{aligned}
2 v\left\langle\mathbf{g}-\mathbf{T}_{h} \mathbf{n}-\left(\mathbf{u}_{h} \otimes \mathbf{u}_{h}\right) \mathbf{n}, \hat{\mathbf{v}}_{h}\right\rangle_{\Gamma_{N}}= & -2 v\left(\mathbf{f}+\operatorname{div} \mathbf{T}_{h}, \hat{\mathbf{v}}_{h}\right)_{\Omega}-2 v\left(\mathbf{T}_{h}, \boldsymbol{\omega}\left(\hat{\mathbf{v}}_{h}\right)\right)_{\Omega} \\
& -\kappa_{2}\left(\mathbf{T}_{h}^{\mathrm{d}}-2 v \mathbf{e}\left(\mathbf{u}_{h}\right)+\left(\mathbf{u}_{h} \otimes \mathbf{u}_{h}\right)^{\mathrm{d}}, \mathbf{e}\left(\hat{\mathbf{v}}_{h}\right)\right)_{\Omega} .
\end{aligned}
$$

Then, combining the latter with the definition of $\mathscr{R}_{2}$ (cf. $(5.10)$ ), and using the fact that $\left(\mathbf{T}_{h}, \boldsymbol{\omega}(\mathbf{z})\right)_{\Omega}=$ $\left(\mathbf{T}_{h}^{\mathrm{d}}, \boldsymbol{\omega}(\mathbf{z})\right)_{\Omega}$ and $\left(-2 v \mathbf{e}\left(\mathbf{u}_{h}\right)+\left(\mathbf{u}_{h} \otimes \mathbf{u}_{h}\right)^{\mathrm{d}}, \boldsymbol{\omega}(\mathbf{z})\right)_{\Omega}=0$ for any $\mathbf{z}$, we observe that $\mathscr{R}_{2}(\mathbf{v})$ can be expressed as follows:

$$
\begin{aligned}
\mathscr{R}_{2}(\mathbf{v})= & 2 v\left(\mathbf{f}+\operatorname{div} \mathbf{T}_{h}, \mathbf{v}-\hat{\mathbf{v}}_{h}\right)_{\Omega}+\kappa_{2}\left(\mathbf{T}_{h}^{\mathrm{d}}-2 v \mathbf{e}\left(\mathbf{u}_{h}\right)+\left(\mathbf{u}_{h} \otimes \mathbf{u}_{h}\right)^{\mathrm{d}}, \mathbf{e}\left(\mathbf{v}-\hat{\mathbf{v}}_{h}\right)_{\Omega}\right. \\
& +2 v\left(\mathbf{T}_{h}^{\mathrm{d}}-2 v \mathbf{e}\left(\mathbf{u}_{h}\right)+\left(\mathbf{u}_{h} \otimes \mathbf{u}_{h}\right)^{\mathrm{d}}, \boldsymbol{\omega}\left(\mathbf{v}-\hat{\mathbf{v}}_{h}\right)\right)_{\Omega}+2 v\left\langle\mathbf{g}-\mathbf{T}_{h} \mathbf{n}-\left(\mathbf{u}_{h} \otimes \mathbf{u}_{h}\right) \mathbf{n}, \mathbf{v}-\hat{\mathbf{v}}_{h}\right\rangle_{\Gamma_{N}} .
\end{aligned}
$$

Therefore, noticing that the boundedness of $\mathbf{I}_{h}$ (Ern \& Guermond 2004 Lemma 1.127, p. 69) implies

$$
\left\|\mathbf{e}\left(\mathbf{v}-\hat{\mathbf{v}}_{h}\right)\right\|_{0, \Omega} \leqslant C\|\mathbf{v}\|_{1, \Omega} \text { and }\left\|\boldsymbol{\omega}\left(\mathbf{v}-\hat{\mathbf{v}}_{h}\right)\right\|_{0, \Omega} \leqslant C\|\mathbf{v}\|_{1, \Omega},
$$

using the Cauchy-Schwarz inequality, estimates (5.14) and (5.15) (for the first and last terms in (5.17)), the fact that the numbers of triangles (tetrahedra) in $\Delta(K)$ and $\Delta(e)$ are bounded, and recalling that $\mathbf{g} \in \mathbf{L}^{2}\left(\Gamma_{N}\right)$, from 5.17) we obtain 5.16, which concludes the proof.

We finally observe that reliability (cf. (5.4) is a direct consequence of Lemmas 5.1 and 5.2

\subsection{Efficiency of the a posteriori error estimator}

TheOREM 5.2 Let $(\mathbf{T}, \mathbf{u}) \in \mathbf{X}$ and $\left(\mathbf{T}_{h}, \mathbf{u}\right) \in \mathbf{X}_{h}$ be the unique solutions of the continuous and discrete problems 3.10 and 4.3, respectively, and assume that $\mathbf{g}$ is piecewise polynomial. Then, there exists $C_{\text {eff }}>0$, independent of $h$, such that

$$
C_{\text {eff }} \Theta \leqslant\left\|\left(\mathbf{T}-\mathbf{T}_{h}, \mathbf{u}-\mathbf{u}_{h}\right)\right\|_{\mathbf{X}} .
$$

To prove Theorem 5.2, in what follows we make extensive use of the original system of equations 2.8, which is recovered from the augmented continuous formulation 3.10 by choosing suitable test functions and integrating by parts backwardly the corresponding equations. We begin with the estimates of the zero order terms appearing in the definition of $\Theta_{K}$ (cf. [5.2).

LEMMA 5.3 There hold

and

$$
\left\|\mathbf{f}+\operatorname{div} \mathbf{T}_{h}\right\|_{0, K} \leqslant\left\|\mathbf{T}-\mathbf{T}_{h}\right\|_{\operatorname{div}, K} \quad \forall K \in \mathscr{T}_{h}
$$

$$
\sum_{K \in \mathscr{T}_{h}}\left\|\mathbf{T}_{h}^{\mathrm{d}}-2 v \mathbf{e}\left(\mathbf{u}_{h}\right)+\left(\mathbf{u}_{h} \otimes \mathbf{u}_{h}\right)^{\mathrm{d}}\right\|_{0, K}^{2} \leqslant C_{1}\left\{\left\|\mathbf{T}-\mathbf{T}_{h}\right\|_{\mathbf{d i v}, \Omega}^{2}+\left\|\mathbf{u}-\mathbf{u}_{h}\right\|_{1, \Omega}^{2}\right\}
$$

where $C_{1}>0$ is independent of $h$.

Proof. For the first term it suffices to recall that $\operatorname{div} \mathbf{T}=-\mathbf{f}$ in $\Omega$ whereas for the second one we use the relation $\mathbf{T}^{\mathrm{d}}-2 v \mathbf{e}(\mathbf{u})+(\mathbf{u} \otimes \mathbf{u})^{\mathrm{d}}=\mathbf{0}$ in $\Omega$, the Cauchy-Schwarz inequality, and estimates 3.19 and 4.28 .

In order to derive the estimate for the third term appearing in $\Theta$ (cf. 5.2), in what follows we make use of an inverse inequality and the localization technique based on edge-bubble or face-bubble functions. To this end, we now introduce further notations and preliminary results. Given $K \in \mathscr{T}_{h}$ and $e \in \mathscr{E}(K)$, we let $\phi_{e}$ be the usual edge-bubble or face-bubble function (Verfürth 1996), which satisfies $\left.\phi_{e}\right|_{K} \in P_{2}(K)$, supp $\phi_{e} \subseteq \omega_{e}:=$ $\cup\left\{K^{\prime} \in \mathscr{T}_{h}: e \in \mathscr{E}\left(K^{\prime}\right)\right\}, \phi_{e}=0$ on $\partial K \backslash e$ and $0 \leqslant \phi_{e} \leqslant 1$ in $\omega_{e}$. We also recall from Verfürth (1994) that, given $k \in \mathbb{N} \cup\{0\}$, there exists an extension operator $L: C(e) \rightarrow C(K)$ satisfying $L(p) \in P_{k}(K)$ and $\left.L(p)\right|_{e}=p$ $\forall p \in P_{k}(e)$. A corresponding vector version of $L$, that is the componentwise application of $L$, is denoted by $\mathbf{L}$. Additional properties of $\phi_{e}$ and $L$ are collected in the following lemma. 
LEMMA 5.4 Given $k \in \mathbb{N} \cup\{0\}$, there exist positive constant $c_{1}$ and $c_{2}$, depending only on $k$ and the shape regularity of the triangulations (minimum angle condition), such that for each $K \in \mathscr{T}_{h}$ and $e \in \mathscr{E}(K)$, there hold

$$
\begin{aligned}
&\|q\|_{0, e}^{2} \leqslant c_{1}\left\|\phi_{e}^{1 / 2} q\right\|_{0, e}^{2} \quad \forall q \in P_{k}(e), \\
&\left\|\phi_{e} L(q)\right\|_{0, K}^{2} \leqslant\left\|\phi_{e}^{1 / 2} L(q)\right\|_{0, K}^{2} \leqslant c_{2} h_{e}\|q\|_{0, e}^{2} \quad \forall q \in P_{k}(e) .
\end{aligned}
$$

Proof. See Lemma 1.3 in Verfürth (1994).

The aforementioned inverse estimate to be utilized next is established now. For its proof we refer the reader to (Ciarlet, 1978, Theorem 3.2.6).

Lemma 5.5 Let $k, l, m \in \mathbb{N} \cup\{0\}$ such that $l \leqslant m$. Then, there exists $c>0$, depending only on $k, l m$ and the shape regularity of the triangulations, such that for each $K \in \mathscr{T}_{h}$ there holds

$$
|q|_{m, K} \leqslant c h_{T}^{l-m}|q|_{l, K}, \quad \forall q \in P_{k}(K) .
$$

Now, we provide the final estimate.

LEMma 5.6 Assume that $\mathbf{g}$ is piecewise polynomial. Then, there exists $C_{2}>0$, independent of $h$, such that

$$
\sum_{e \in \mathscr{E}\left(\Gamma_{N}\right)} h_{e}\left\|\mathbf{g}-\mathbf{T}_{h} \mathbf{n}-\left(\mathbf{u}_{h} \otimes \mathbf{u}_{h}\right) \mathbf{n}\right\|_{0, e}^{2} \leqslant C_{2}\left\{\sum_{e \in \mathscr{E}\left(\Gamma_{N}\right)}\left\|\mathbf{T}-\mathbf{T}_{h}\right\|_{\mathbf{d i v}, K_{e}}^{2}+h\left\|\mathbf{u}-\mathbf{u}_{h}\right\|_{1, \Omega}^{2}\right\},
$$

for all $e \in \mathscr{E}_{h}\left(\Gamma_{N}\right)$, where $K_{e}$ is the triangle of $\mathscr{T}_{h}$ having $e$ as an edge.

Proof. Given $e \in \mathscr{E}\left(\Gamma_{N}\right)$, we let $K_{e}$ be the element of $\mathscr{T}_{h}$ having $e$ as an edge or face, and define $\mathbf{v}_{e}:=$ $\mathbf{g}-\mathbf{T}_{h} \mathbf{n}-\left(\mathbf{u}_{h} \otimes \mathbf{u}_{h}\right) \mathbf{n}$ on $e$. Then, we proceed similarly to the proof of (Gatica et al. 2011c. Lemma 3.16), that is, we apply (5.19), recall that $\phi_{e}=0$ on $\partial K_{e} \backslash e$, extend $\phi_{e} \mathbf{L}\left(\mathbf{v}_{e}\right)$ by zero in $\Omega \backslash K_{e}$ so that the resulting function belongs to $\mathbf{H}^{1}(\Omega)$, use that $\mathbf{g}=\mathbf{T} \mathbf{n}+(\mathbf{u} \otimes \mathbf{u}) \mathbf{n}$ on $\Gamma_{N}$, integrate by parts in $\Omega$, and make use of 5.20 , and 5.21 , to arrive at

$$
\left\|\mathbf{v}_{e}\right\|_{0, e} \leqslant C h_{e}^{1 / 2}\left\{h_{T_{e}}^{-1}\left\|\mathbf{T}-\mathbf{T}_{h}\right\|_{0, K_{e}}+\left\|\operatorname{div}\left(\mathbf{T}-\mathbf{T}_{h}\right)\right\|_{0, K_{e}}\right\}+C\left\|(\mathbf{u} \otimes \mathbf{u}) \mathbf{n}-\left(\mathbf{u}_{h} \otimes \mathbf{u}_{h}\right) \mathbf{n}\right\|_{0, e},
$$

which implies

$$
h_{e}\left\|\mathbf{v}_{e}\right\|_{0, e}^{2} \leqslant C\left\{\left\|\mathbf{T}-\mathbf{T}_{h}\right\|_{\mathbf{d i v}, K_{e}}^{2}+h_{e}\left\|(\mathbf{u} \otimes \mathbf{u}) \mathbf{n}-\left(\mathbf{u}_{h} \otimes \mathbf{u}_{h}\right) \mathbf{n}\right\|_{0, e}^{2}\right\} .
$$

In turn, using estimates 3.3, 3.35, 4.15) and the fact that $\mathbf{H}^{1 / 2}(\Gamma)$ is continuously embedded into $\mathbf{L}^{4}(\Gamma)$, and proceeding similarly as in 4.28 , we easily obtain that

$$
\sum_{e \in \mathscr{E}\left(\Gamma_{N}\right)}\left\|(\mathbf{u} \otimes \mathbf{u}) \mathbf{n}-\left(\mathbf{u}_{h} \otimes \mathbf{u}_{h}\right) \mathbf{n}\right\|_{0, e}^{2}=\left\|(\mathbf{u} \otimes \mathbf{u}) \mathbf{n}-\left(\mathbf{u}_{h} \otimes \mathbf{u}_{h}\right) \mathbf{n}\right\|_{0, \Gamma_{N}}^{2} \leqslant C\left\|\mathbf{u}-\mathbf{u}_{h}\right\|_{1, \Omega}^{2} .
$$

From this estimate, 5.24, and the fact that $h_{e} \leqslant h$, we obtain 5.22) which concludes the proof.

We conclude by observing that the efficiency of $\Theta$ follows straightforwardly from Lemmas 5.3 and 5.6

\section{Numerical results}

The subsequent examples serve to test the performance of the proposed scheme, to confirm the convergence rates anticipated by Theorem 4.3 . and to illustrate the efficiency and reliability of the a posteriori error estimators.

Test 1. In the first example we consider $n=2$ and manufacture the following exact solutions to [2.8 with mixed boundary conditions, defined on the rectangular domain $\Omega=(0,3 / 2) \times(0,1)$

$$
\mathbf{u}=\left(\begin{array}{c}
-2 x^{2}(x-1)^{2} y(y-1)(2 y-1) \\
2 y^{2}(y-1)^{2} x(x-1)(2 x-1)
\end{array}\right), \quad p=x^{3}-y^{4}-\frac{1.5^{4}}{4}+\frac{1}{5}, \quad \mathbf{T}=2 v \mathbf{e}(\mathbf{u})-\mathbf{u} \otimes \mathbf{u}-p \mathbf{I} .
$$

These functions satisfy the analyzed regularity and are employed to set up the external load $\mathbf{f}$, and the nonhomogeneous boundary traction $\mathbf{g}$ defined on the right wall of the rectangle, which constitutes $\Gamma_{N}$. Notice that the exact displacements vanish on $\Gamma_{D}$ (conformed by the bottom, top, and left edges of the domain). The fluid viscosity is taken as $v=1$ and the augmentation constants are set as $\kappa_{1}=\kappa_{2}=2 v=2$. Following the structure of the linearization used to establish the solvability of both continuous and discrete problems, here we employ 
TABLE 2. Test 1A: errors, convergence rates, effectivity index associated to the a posteriori error indicator, and Picard iteration count for the $\mathbf{R T}_{k}-P_{k+1}$ approximation of the Navier-Stokes equations in mixed form. The error history for the post-processed pressure (projected onto the space of discontinuous $P_{k+2}$ elements) is also displayed.

\begin{tabular}{rccccccccc}
\hline Dof & $h$ & $e(\mathbf{T})$ & rate & $e(\mathbf{u})$ & rate & $e(p)$ & rate & eff $(\theta)$ & Iter \\
\hline \multicolumn{8}{c}{$k=0$} \\
\hline 70 & 0.7071 & 1.8092 & - & 0.2599 & - & 0.4709 & - & 0.9736 & 4 \\
126 & 0.5017 & 1.3970 & 0.7534 & 0.2258 & 0.4108 & 0.3515 & 0.8519 & 1.0000 & 5 \\
330 & 0.2931 & 0.8680 & 0.8854 & 0.1546 & 0.7040 & 0.2069 & 0.9861 & 1.0207 & 5 \\
1026 & 0.1601 & 0.4868 & 0.9569 & 0.0899 & 0.8970 & 0.1153 & 0.9673 & 1.0540 & 5 \\
3570 & 0.0840 & 0.2577 & 0.9857 & 0.0479 & 0.9741 & 0.0608 & 0.9902 & 1.0573 & 5 \\
13266 & 0.0430 & 0.1325 & 0.9956 & 0.0246 & 0.9957 & 0.0311 & 1.0015 & 1.0676 & 5 \\
51090 & 0.0218 & 0.0671 & 0.9986 & 0.0124 & 0.9996 & 0.0157 & 1.0021 & 1.0677 & 5 \\
200466 & 0.0109 & 0.0338 & 0.9995 & 0.0062 & 1.0000 & 0.0079 & 1.0012 & 1.0700 & 5 \\
\hline & & \multicolumn{8}{c}{$k=1$} \\
\hline 10 & 0.7071 & 0.5802 & - & 0.1341 & - & 0.0786 & - & 0.9579 & 5 \\
394 & 0.5017 & 0.3287 & 1.6555 & 0.0780 & 1.5796 & 0.0476 & 1.4608 & 0.9611 & 5 \\
1078 & 0.2931 & 0.1236 & 1.8195 & 0.0308 & 1.7273 & 0.0185 & 1.7541 & 0.9676 & 5 \\
3454 & 0.1601 & 0.0384 & 1.9348 & 0.0098 & 1.8819 & 0.0058 & 1.9043 & 0.9987 & 5 \\
12238 & 0.0840 & 0.0107 & 1.9756 & 0.0028 & 1.9441 & 0.0016 & 1.9424 & 1.0107 & 5 \\
45934 & 0.0430 & 0.0028 & 1.9900 & 0.0007 & 1.9718 & 0.0004 & 1.9620 & 1.0228 & 5 \\
177838 & 0.0218 & 0.0007 & 1.9955 & 0.0002 & 1.9856 & 0.0001 & 1.9774 & 1.0308 & 5 \\
699694 & 0.0109 & 0.0001 & 1.9979 & 0.0001 & 1.9926 & $3.0 \mathrm{e}-5$ & 1.9876 & 1.0402 & 5 \\
\hline
\end{tabular}

a fixed point algorithm, and at each Picard iteration the linear systems are solved with the multifrontal direct solver MUMPS. We proceed to study the accuracy of the finite element schemes of order $k \in\{0,1\}$ by solving the discrete problem on a sequence of successively refined triangulations of $\Omega$ and computing errors for stress and velocity in their natural norms. The error history is collected in Table 2 , which indicates that the method converges optimally to the exact solutions. Accordingly, we observe effectivity indexes (defined in 6.1, below) that approach very closely the optimal value 1 . The fixed point iterates are terminated when the residual attains a fixed tolerance of $1 \mathrm{e}-9$, and the table shows that a maximum of five iterations are required. For reference, we also include in Figure 6.1 the flow patterns obtained with the lowest order method on a structured mesh with 50438 elements.

We also investigate the robustness of the formulation with respect to the viscosity value. From the structure of the closed form solutions we observe that both velocity and pressure do not depend on $v$. So we repeat the convergence study above concentrating on the accuracy of the stress approximation and the number of fixed point iterations when we refine the mesh, and when we decrease the viscosity. In Table 3 we observe that smaller viscosities produce a deterioration of the convergence (both in terms of error decay of the stress approximation and iteration count), especially for coarser meshes.

Test 2. For our second numerical example we perform the classical test of flow over a backward-facing step. The problem set up consists of a channel of adimensional length 5 and height 1 , expanding abruptly to a height of 2. Channels shorter than usual can be easily studied since outflow conditions can be readily incorporated with the present formulation. Recirculating flows are expected just after the step, whose shape and size depend on the Reynolds number (in this case, $\operatorname{Re}=D U / v=200$, where $v=0.01$ is the kinematic viscosity, $D=2$ is twice the inlet height and $U=1 / 2$ is the mean inlet velocity). A parabolic inflow velocity profile is imposed on the left part of the domain $\mathbf{u}=\left(2 x_{2}\left(1-x_{2}\right), 0\right)^{T}$, a zero stress condition is set on the right end of the channel (corresponding to consider $\mathbf{g}=\mathbf{0}$ ) and no-slip velocities are set on the remainder of $\partial \Omega$. After constructing a coarse unstructured triangular mesh, the problem is solved using the proposed mixed finite element scheme, the local a posteriori error estimator (5.2) is computed, and elements are marked for refinement according to the classical maximal error strategy. Then a new mesh is generated and the adaptive process is repeated seven times. Numerical results obtained on the finest level are portrayed in the first three rows of Figure 6.2 Wellresolved profiles are obtained for stresses and the velocity components with streamlines show the presence 
TABLE 3. Test 1B: accuracy of the stress approximation and iteration count produced with a lowest-order method, for different viscosity values.

\begin{tabular}{rcccc}
\hline Dof & $e(\mathbf{T})$ & rate & eff $(\theta)$ & Iter \\
\hline \multicolumn{5}{c}{$v=0.1$} \\
10 & 1.3048 & - & 1.1975 & 6 \\
126 & 0.9550 & 0.9095 & 1.2383 & 7 \\
330 & 0.5630 & 0.9829 & 1.2075 & 7 \\
1026 & 0.3082 & 0.9968 & 1.1564 & 7 \\
3570 & 0.1617 & 0.9996 & 1.1286 & 6 \\
13266 & 0.0829 & 1.0001 & 1.1183 & 7 \\
51090 & 0.0419 & 1.0001 & 1.1150 & 7 \\
200466 & 0.0223 & 1.0000 & 1.0536 & 7 \\
\hline \multicolumn{5}{c}{$v=0.01$} \\
\hline 70 & 4.9397 & - & 1.1637 & 11 \\
126 & 4.5050 & 0.1394 & 1.4477 & 11 \\
330 & 3.8334 & 0.4758 & 1.3101 & 12 \\
1026 & 0.6837 & 2.8530 & 1.6875 & 10 \\
3570 & 0.1624 & 2.2279 & 1.9585 & 11 \\
13266 & 0.0814 & 1.0326 & 1.7924 & 11 \\
51090 & 0.0410 & 1.0076 & 1.3378 & 11 \\
200466 & 0.0230 & 1.0021 & 1.1391 & 11 \\
\hline \multicolumn{5}{c}{$v=0.001$} \\
\hline 70 & 11.704 & - & 1.2354 & 15 \\
126 & 7.9093 & 0.4422 & 1.0702 & 17 \\
330 & 5.1204 & 0.7083 & 1.0017 & 15 \\
1026 & 1.0865 & 0.6041 & 1.2241 & 16 \\
3570 & 0.6933 & 0.7980 & 1.8104 & 16 \\
13266 & 0.1320 & 1.3775 & 1.8634 & 16 \\
51090 & 0.0749 & 0.9607 & 0.8227 & 17 \\
200466 & 0.0482 & 0.7691 & 0.9253 & 17 \\
\hline \multicolumn{5}{c}{}
\end{tabular}

of the expected reattaching flow behaviour. The last row of the figure depicts adaptively refined meshes at intermediate steps of the algorithm. They indicate a clear clustering of elements near the reentrant corner of the domain, as well as near the zones of high velocity gradients and stress concentration. Notice that the magnitude of $T_{11}$ dominates all other individual fields, which results in the fact that this quantity also dictates where the mesh refinement is applied. For this example we used a Newton method with a fixed tolerance of $1 \mathrm{e}-7$ and the linear systems were solved with the UMFPACK solver.

Test 3. Next we turn to the testing of the scheme and the adaptive algorithm in a 3D scenario. We want to compute errors produced by our mixed method, satisfying precisely the analyzed boundary conditions, but considering a non-convex domain. We take a computational domain defined as $\Omega=(0,1)^{3} \backslash[0.5,1]^{3}$ and we manufacture the following exact solutions of the Navier-Stokes equations 2.8

$$
\begin{gathered}
\mathbf{u}=\left(\begin{array}{c}
\sin ^{2}\left(\pi x_{1}\right) \sin \left(\pi x_{2}\right) \sin \left(2 \pi x_{3}\right) \\
\sin \left(\pi x_{1}\right) \sin ^{2}\left(\pi x_{2}\right) \sin \left(2 \pi x_{3}\right) \\
-\left[\sin \left(2 \pi x_{1}\right) \sin \left(\pi x_{2}\right)+\sin \left(\pi x_{1}\right) \sin \left(2 \pi x_{2}\right)\right] \sin ^{2}\left(\pi x_{3}\right)
\end{array}\right), \\
\mathbf{T}=2 v \mathbf{e}(\mathbf{u})-\mathbf{u} \otimes \mathbf{u}-\frac{1-x_{1}^{2}-x_{2}^{2}-x_{3}^{2}}{\left(x_{1}-0.55\right)^{2}+\left(x_{2}-0.55\right)^{2}+\left(x_{3}-0.55\right)^{2}} \mathrm{I},
\end{gathered}
$$

which may appear contrived, but their specific form obeys to satisfaction of homogeneous Dirichlet conditions for the velocity on $\Gamma_{D}$ (the sides lying on the unit cube $\left.(0,1)^{3}\right)$, and which we also employ to specify the forcing term and the non-homogeneous normal stress condition on $\Gamma_{N}$ (the remainder of the boundary). Notice that the 

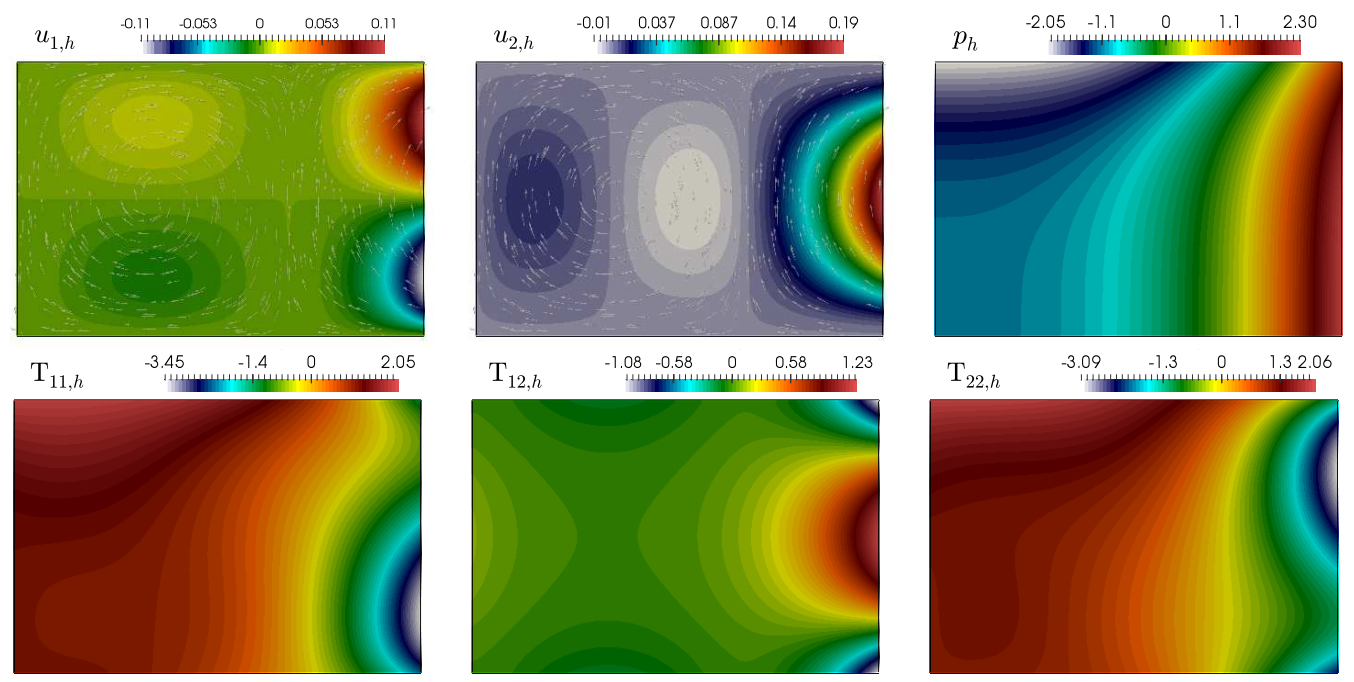

FIG. 6.1. Test 1A: approximate numerical solutions computed with the lowest order method. Velocity components, postprocessed pressure, and total stress components.
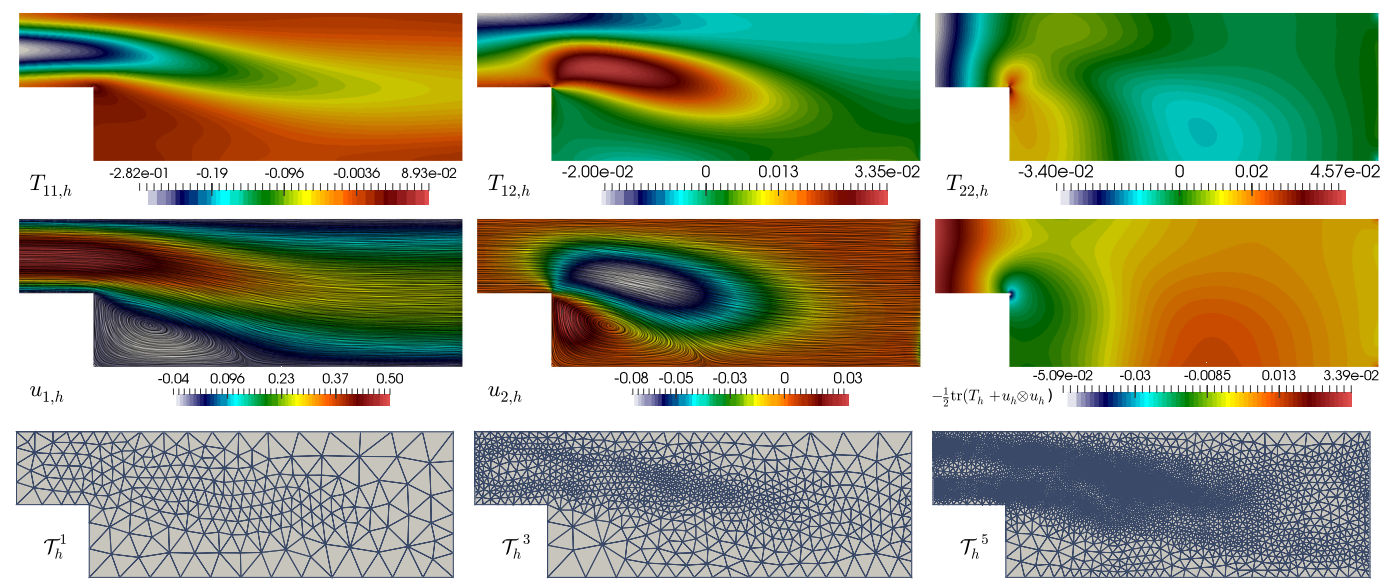

FIG. 6.2. Test 2: approximate numerical solutions of the backward-facing step benchmark, computed with a method of order $k=1$. Total stress components (top panels), velocity components and streamlines and post-processed pressure (middle row), and three snapshots of meshes after one, three, and five adaptive refinement steps according to the a posteriori error estimator (bottom).

singularity of the stress near the reentrant quadrant at $(0.55,0.55,0.55)$ anticipates that the convergence will be affected if uniform refinement is applied. We define the total error and the effectivity index associated to the a posteriori error estimator as

$$
\mathrm{e}:=\left\{[e(\mathbf{T})]^{2}+[e(\mathbf{u})]^{2}\right\}^{1 / 2}, \quad \text { and } \quad \operatorname{eff}(\theta):=\mathrm{e} \theta^{-1},
$$

respectively. The viscosity is taken as $v=1$ and two runs of the error analysis are performed: one with uniform mesh refinement (however, we do not apply a nested refinement, as we only consider partitions with $2 \mathrm{~m}$ points on a given edge, on each refinement step $m=1, \ldots$ ) and one under adaptive mesh refinement based on a distribution of the local error indicators so that the marked elements represent a $70 \%$ of the total error. Table 4 shows the obtained error history, indicating sub-optimal convergence rates and oscillating effectivity indexes for the constantly refined meshes, while both the expected optimal convergence and the steady effectivity indexes are restored under adaptive refinement.

We present in Figure 6.3 examples of approximate solutions along with snapshots of a few adaptive meshes generated following the a posteriori error estimator. Elements are concentrated near the origin and on regions 

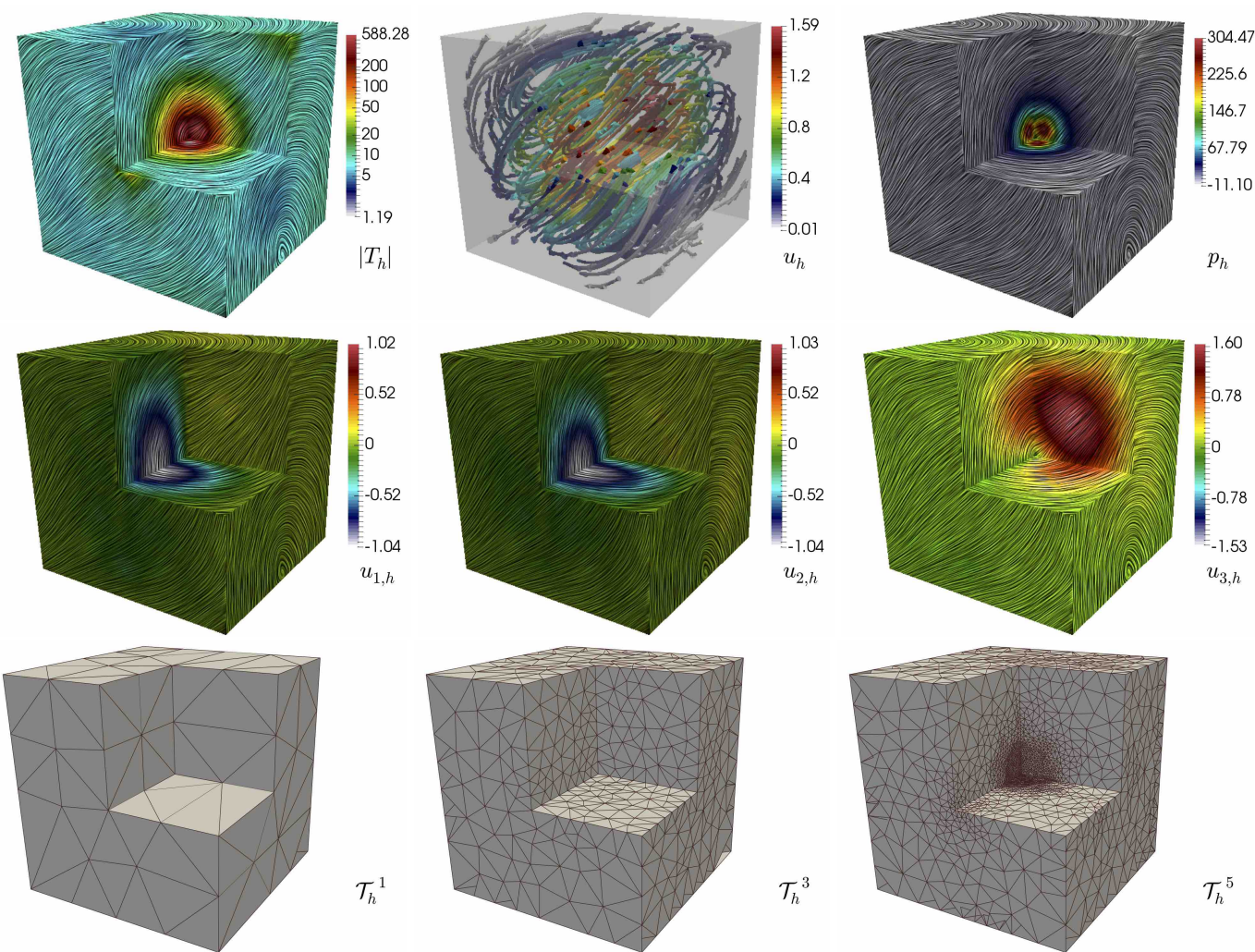

FIG. 6.3. Test 3: approximate numerical solutions computed with a method of order $k=0$. Total stress magnitude, velocity streamlines and post-processed pressure (top panels), velocity components (middle row), and three snapshots of meshes after one, three, and five adaptive refinement steps according to the a posteriori error estimator (bottom).

of high stress, and even with rather coarse meshes, the produced stress and velocity profiles are well-resolved.

Funding. This research was partially supported by CONICYT-Chile through project Inserción de Capital Humano Avanzado en la Academia 79130048; project Fondecyt 11140691 and project Fondecyt 1161325; by Universidad del Bío-Bío through DIUBB project 151408 GI/VC; by the Engineering and Physical Sciences Research Council EPSRC through the research grant EP/R00207X/1 and by MINECO grant MTM2015-69875P (Ministerio de Economa y Competitividad, Spain).

\section{References}

ARnold, D.N., Douglas, J. AND GuPTA, C.P. A family of higher order mixed finite element methods for plane elasticity. Numerische Mathematik, vol. 45, pp. 1-22, (1984).

ARnold, D.N. BREZZI F. AND Douglas JR., J. PEERS: a new mixed finite element for plane elasticity, Japan Journal of Applied Mathematics, vol. 1, no. 2, pp. 347-367, (1984)

B ÄNSCH, E. Finite element discretization of the Navier-Stokes equations with a free capillary surface. Numerische Mathematik, vol. 88, no. 2, pp. 203-235, (2001).

B ÄNSCH, E. A finite element pressure correction scheme for the Navier-Stokes equations with traction boundary condition. Computer Methods in Applied Mechanics and Engineering, vol. 279, pp. 198-211, (2014).

BRUNEAU, C.-H. AND FABRIE, P. New efficient boundary conditions for incompressible Navier-Stokes equations: a well-posedness result. Modélisation Mathématique et Analyse Numérique, vol. 30, no. 7, pp. 815840, (1996). 
TABLE 4. Test 3: errors, convergence rates, effectivity indexes, and fixed point steps to converge for the $\mathbf{R T}_{0}-P_{1}$ approximation of the Navier-Stokes equations in mixed form under uniform non-nested (top rows) and adaptive (bottom rows) refinement according to the proposed a posteriori error estimator.

\begin{tabular}{rccccccccc}
\hline Dof & $h$ & $e(\mathbf{T})$ & rate & $e(\mathbf{u})$ & rate & $e(p)$ & rate & eff $(\theta)$ & Iter \\
\hline \multicolumn{8}{c}{ uniform, non-nested refinement } \\
\hline 402 & 0.7071 & 161.4696 & - & 4.5341 & - & 161.5333 & - & 0.9548 & 3 \\
2655 & 0.3536 & 148.2176 & 0.0907 & 3.2936 & 0.3387 & 148.2542 & 0.0909 & 0.8627 & 5 \\
8400 & 0.2357 & 130.4515 & 0.2217 & 2.5345 & 0.4549 & 130.4762 & 0.2218 & 0.8820 & 5 \\
19275 & 0.1768 & 128.6967 & 0.1092 & 2.0552 & 0.5047 & 128.7125 & 0.1090 & 0.8237 & 6 \\
36918 & 0.1414 & 121.6633 & 0.2903 & 1.7212 & 0.5458 & 121.6754 & 0.2903 & 0.9037 & 5 \\
62967 & 0.1179 & 110.4270 & 0.3630 & 1.4844 & 0.5544 & 110.4370 & 0.3630 & 0.8644 & 5 \\
146835 & 0.0884 & 93.9559 & 0.4198 & 1.1782 & 0.5399 & 93.9633 & 0.4198 & 0.9734 & 6 \\
\hline \multicolumn{8}{c}{ adaptive refinement } \\
\hline 402 & 0.7071 & 161.4696 & - & 4.5341 & - & 161.5333 & - & 0.9548 & 3 \\
1575 & 0.6270 & 106.6375 & 0.8559 & 2.0084 & 0.8805 & 106.7010 & 0.8557 & 0.9638 & 5 \\
9000 & 0.3138 & 87.0510 & 0.9215 & 1.0573 & 0.9493 & 87.0769 & 0.9211 & 0.9863 & 5 \\
40629 & 0.2789 & 60.8049 & 0.9397 & 0.8174 & 0.9531 & 60.8231 & 0.9396 & 0.9878 & 5 \\
130518 & 0.2608 & 40.8514 & 0.9936 & 0.5849 & 0.9346 & 40.8761 & 0.9932 & 0.9904 & 5 \\
413919 & 0.2500 & 17.1690 & 1.0186 & 0.3311 & 1.0598 & 17.2243 & 1.0149 & 0.9857 & 5 \\
\hline
\end{tabular}

Brezzi, F. AND Fortin, M. Mixed and Hybrid Finite Element Methods. Springer Series in Computational Mathematics, 15. Springer-Verlag, New York, 1991.

CAI, Z. WANG, C. AND ZHANG, S. Mixed finite element methods for incompressible flow: stationary NavierStokes equations. SIAM Journal on Numerical Analysis, vol 48, no. 1, pp. 79-94, (2010).

CAI, Z. AND ZHANG, S. Mixed methods for stationary Navier-Stokes equations based on pseudostresspressure-velocity formulation. Mathematics of Computation, vol. 81, no. 280, pp. 1903-1927, (2012).

Camaño, J., Gatica, G.N., Oyarzúa, R., Ruiz-Baier, R. And Venegas, P. New fully-mixed finite element methods for the Stokes-Darcy coupling. Computer Methods in Applied Mechanics and Engineering, vol. 295, pp. 362-395, (2015).

CAMAÑo, J., OYARzÚA, R. AND TIERRA, G. Analysis of an augmented mixed-FEM for the Navier-Stokes problem. Mathematics of Computation, vol. 86, no. 304, pp. 589-615, (2017).

CAMAÑo, J., GATICA, G.N., OYARZÚA, R. AND TIERRA, G. An augmented mixed finite element method for the Navier-Stokes equations with variable viscosity. SIAM Journal on Numerical Analysis, vol. 54, 2, pp. 1069-1092, (2016).

Caucao, S. Gatica, G.N., Oyarzúa, R. and Sebestová, I. A fully-mixed finite element method for the Navier-Stokes/Darcy coupled problem with nonlinear viscosity. Journal of Numerical Mathematics, to appear. DOI: 10.1515/jnma-2015-0121.

Ciarlet, P.G. The finite Element Method for Elliptic Problems. North-Holland, Amsterdam, New York, Oxford, (1978).

CLÉment, P. Approximation by finite element functions using local regularisation. RAIRO Modélisation Mathématique et Analyse Numérique, vol. 9, pp. 77-84, (1975).

COlmenares, E., GaticA, G.N. And OYARZÚA, R. Analysis of an augmented mixed-primal formulation for the stationary Boussinesq problem. Numerical Methods for Partial Differential Equations, vol. 32, no. 2, pp. 445-478, (2016).

DONG, S. AND SHEN, J. A pressure correction scheme for generalized form of energy-stable open boundary conditions for incompressible flows. Journal of Computational Physics, vol. 291, pp. 254-278, (2015). 
Ern, A. And Guermond, J.-L. Theory and Practice of Finite Elements. Applied Mathematical Sciences, Springer-Verlag, New York, vol. 159, (2004).

ERVin, V.J., Howell, J.S. AND STANCUlescu, I. A dual-mixed approximation method for a three-field model of a nonlinear generalized Stokes problem. Computer Methods in Applied Mechanics and Engineering, vol. 197, 33-40, pp. 2886-2900, (2008).

FARHLOUl, M. AND ForTin, M. Dual hybrid methods for the elasticity and the Stokes problems: a unified approach. Numerische Mathematik, vol. 76, pp. 419-440, (1997).

Farhloul, M., Nicaise, S. And PaQuet, L. A refined mixed finite-element method for the stationary Navier-Stokes equations with mixed boundary conditions. IMA Journal of Numerical Analysis, vol. 28, no. 1, pp. 25-45, (2008).

FARhloul, M., NiCAise, S. And PAQUet, L. A priori and a posteriori error estimations for the dual mixed finite element method of the Navier-Stokes problem. Numerical Methods for Partial Differential Equations, vol. 25 , no. 4, pp. 843-869, (2009).

Franca, L.P. And Hughes, T.J.R. Two classes of mixed finite element methods. Computer Methods in Applied Mechanics and Engineering, vol. 69, no. 1, pp. 89-129, (1988).

GATICA, G.N. Analysis of a new augmented mixed finite element method for linear elasticity allowing $\mathrm{RT}_{0-}$ $P_{1}-P_{0}$ approximations. ESAIM: Mathematical Modelling and Numerical Analysis, vol. 40, no. 1, pp. 1-28, (2006).

GaticA, G.N. A Simple Introduction to the Mixed Finite Element Method. Theory and Applications. Springer Briefs in Mathematics, Springer, Cham Heidelberg New York Dordrecht London, (2014).

GATICA, G.N., OYARZÚA, R. AND SAYAs, F.J. Analysis of fully-mixed finite element methods for the StokesDarcy coupled problem. Mathematics of Computation, vol. 80, no. 276, pp. 1911-1948, (2011).

Gatica, G.N., OYARZÚA, R. AND SAYAS, F.J. A residual-based a posteriori error estimator for a fullymixed formulation of the Stokes-Darcy coupled problem. Computer Methods in Applied Mechanics and Engineering, vol. 200, pp. 1877-1891, (2011).

GATICA, G.N., OYARZÚA, R. AND SAYAS, F.J. A twofold saddle point approach for the coupling of fluid flow with nonlinear porous media flow. IMA Journal of Numerical Analysis, vol. 32, no. 3, pp. 845-887, (2012).

GaticA, G.N. RUIZ-BAIER, R. AND TIERRA, G. A posteriori error analysis of an augmented mixed method for the Navier-Stokes equations with nonlinear viscosity. Computers \& Mathematics with Applications, vol. 72, no. 9, pp. 2289-2310, (2016).

Girault, V. And RaViart, P.-A. Finite element methods for Navier-Stokes equations. Theory and algorithms. Springer Series in Computational Mathematics, 5. Springer-Verlag, Berlin (1986).

Howell, J.S. AND Walkington, N. Dual mixed finite element methods for the Navier-Stokes equations. ESAIM: Mathematical Modelling and Numerical Analysis, vol. 47, pp. 789-805, (2013).

LEE, S. AND SALGADO, A.J. Stability analysis of pressure correction schemes for the Navier-Stokes equations with traction boundary conditions. Computer Methods in Applied Mechanics and Engineering, vol. 309, pp. 307-324, (2016).

VERFÜRTH, R. A Review of A Posteriori Error Estimation and Adaptive Mesh-Refinement Techniques. WileyTeubner, Chichester, (1996).

VERFÜRTH, R. A posteriori error estimation and adaptive mesh-refinement techniques. Journal of Computational and Applied Mathematics, vol. 50, pp. 67-83, (1994). 